\title{
Dimensionen der Wahrnehmung von Varianz
}

\author{
Joachim Gessinger (Potsdam)
}

\begin{abstract}
The SiN-project data corpus delivers an empirical foundation for the study of different dimensions in the perception of variation as "divergence from common usage" (H. Paul). In addition to the "objective" linguistic data (Produktionsdaten) obtained through biographical interviews, informal chats with relations and friends (Tischgespräche) as well as tasks to explore the variational repertoire of the informants, the corpus also displays results of the elicitation of linguistic knowledge and language attitudes obtained through specific testing methods, in which the purpose was to evaluate non-standard language features as to their salience, the situational dependence and norm adequacy and to trigger metalinguistic comments. In order to give plausible qualitative and quantitative evidence about the regional diversity of perception and linguistic variation in Northern Germany I will show the interdependence between the realization of phonological features divergent from spoken standard and perceptional dispositions of the informants which indicate the sociopragmatic function of phonological and morphosyntactic variation.
\end{abstract}

\section{$1 \quad$ SiN-Korpus}

Das Projekt „Sprachvariation in Norddeutschland“ (SiN) ist in zahlreichen Publikationen vorgestellt worden, zuletzt im Kontext anderer variationslinguistischer Forschungen zum Deutschen im Sammelband von Kehrein/Lameli/Rabanus (2015). ${ }^{.}$Die Forschergruppe hat es sich zum Ziel gesetzt, mit unterschiedlichen variations-, perzeptions- und soziolinguistischen Methoden die rezenten Sprachverhältnisse im norddeutschen Raum zu untersuchen und mit dieser Momentaufnahme die strukturellen Veränderungen im Varietätengefüge dieses Raums zu dokumentieren.

Ein erstes Ergebnis liegt seit 2015 vor: Der erste von zwei Atlasbänden (NOSA), der die räumliche und quantitative Verteilung von für Norddeutschland relevanten phonologischen und morphosyntaktischen hochdeutsch basierten Variablen zeigt und ausführlich kommentiert (cf. Elmentaler/Rosenberg 2015). Wer sich die Kommentare anschaut, wird nachvollziehen können, wieviel Potential in der systematischen Zusammenführung von den häufig als „objektiv“ deklarierten Produktionsdaten und „subjektiv“ genannten Wahrnehmungsdaten steckt. Die Fragestellungen der Teilprojekte, das Zusammenspiel der unterschiedlichen Erhebungs-

${ }^{1}$ Cf. Elmentaler et al. 2015.

Linguistik online 85, 6/17 - http://dx.doi.org/10.13092/lo.85.4080

CC by 3.0 
methoden und die Möglichkeiten, unterschiedliche Datentypen aufeinander zu beziehen, werden in folgendem Schema deutlich:

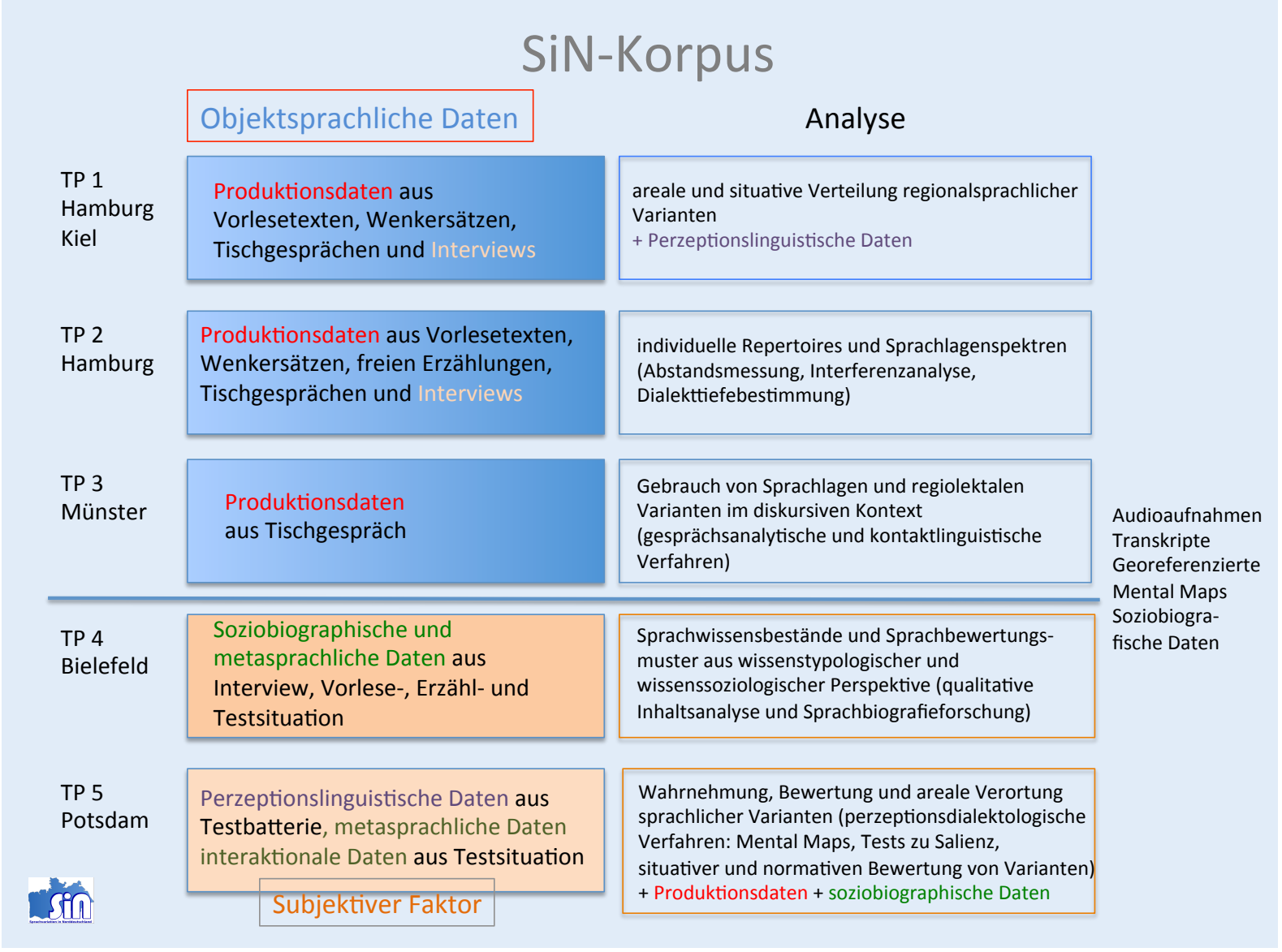

Abbildung 1: SiN-Korpus

Der Unterschied zwischen den beiden empirisch gewonnen Datentypen liegt nicht in ihrer Objektivität, sondern in der Perspektive, aus der sie erzeugt und analysiert werden: Beide Datentypen sind Äußerungen von Gewährspersonen (GP). Die Produktionsdaten sind objektsprachliche Daten der GP, die im Projekt transkribiert und analysiert werden, um Gebrauchsnormen von Varianten in ihrer individuellen, situativen und arealen Ausprägung und die diskursive Verwendung von Varianten und Sprachlagen zu rekonstruieren (Teilprojekte 1, 2 und 3). Dabei werden neben qualitativen und quantitativen Methoden der Variationslinguistik auch kontaktlinguistische und gesprächsanalytische Verfahren eingesetzt. Die Daten zur Wahrnehmung, Klassifikation und Bewertung von Sprachdaten (Teilprojekt 5) werden in mehreren, aufeinander bezogenen perzeptionslinguistisch basierten Verfahren gewonnen, wobei die dabei erzeugten objektsprachlichen Daten vor allem auf ihre soziopragmatischen und teilweise auch interaktionalen Aspekte hin untersucht werden.

Ich fasse die Daten aus Teilprojekt 5 unter das Label „subjektiver Faktor“, weil sie die normative, situative und kognitive Dimension der sprachlichen und nonverbalen Äußerungen der GP beobachtbar machen und Rückschlüsse auf jene sozialevaluativen Instanzen erlauben, die Äußerungen steuern und sprachlichen Wandel motivieren.

Ein dritter Datentyp, auf den ich hier nicht weiter eingehen werde, sind soziobiographische Daten und Äußerungen der GP im leitfadengesteuerten Interview und in freien Erzählungen (Teilprojekt 4). Sie gehen zum einen als objektsprachliche Daten in die Analyse der Produkti- 
onsdaten ein, zum anderen werden sie als sich in diesen Äußerungen artikulierende, unterschiedlich strukturierte Wissensbestände der GP aus wissenstypologischer und wissenssoziologischer Perspektive untersucht. ${ }^{2}$

Diese Daten mit den für die Analyse notwendigen Annotationen bilden das SiN-Korpus. An einem kleinen Ausschnitt am Beispiel der $g$-Spirantisierung im Morphemauslaut möchte ich Möglichkeiten (und Grenzen) der im Projekt angewandten Methoden zeigen.

Insgesamt sechs Non-Standard-Varianten wurden in den Perzeptionstests des Teilprojekts 5 (s. Abb. 1) für alle Erhebungsregionen abgefragt, ihre jeweiligen Merkmale sind als Variablen im NOSA 1 beschrieben: gefracht, Pabbe, Kinner, Kaffe, Kopp und dat. Die Spirantisierung von - $g$ im Morphemauslaut wie in gefracht gilt wie andere Spirantisierungen als ein norddeutsches Merkmal. ${ }^{3}$ Es ist in der Literatur ausgiebig besprochen und hat im NOSA 1 einen der beiden Herausgeber, Peter Rosenberg, zu einem der vermutlich umfangreichsten Kommentare des Bandes provoziert. Die initiale Spirantisierung $j$ - wie in janz und jemacht wurde in den Perzeptionstests nur in einigen Regionen (Brandenburg, Niederrhein und Mittelpommern) abgefragt, es gibt aber, soviel sei hier schon angedeutet, einen Zusammenhang im Auftreten von initialer und finaler Spirantisierung.

\section{Produktionsdaten zur $\mathbf{g}$-Spirantisierung im Morphemauslaut}

Die folgende Tabelle aus NOSA 1 (Elmentaler/Rosenberg 2015: 267) gibt eine Übersicht über die Frequenz der finalen $g$-Spirantisierungen in unterschiedlichen phonologischen Umgebungen in den Produktionsdaten:

\footnotetext{
2 Dazu Elmentaler et al. (2015: 418f.).

3 Cf. u. a. Stellmacher (1977), zur aktuellen Situation in Schleswig-Holstein und Mecklenburg-Vorpommern Scharioth (2015). So wird schon im Kontext des Schreibsprachenwechsels zum Hochdeutschen, wie Lambecks Düedsche Orthographia (Hamburg 1633) zeigt, auf das Problem der Homophonie von Dach und Tag hingewiesen, die für eine korrekte Schreibung von $<$ Tag $>$ hinderlich sei, was darauf hindeutet, dass der schriftsprachliche Druck auf die $g$-Spirans auf Dauer zu ihrer Schwächung geführt haben dürfte. Viëtor weist im Vorwort der 4. Aufl. seiner Aussprache des Schriftdeutschen (1898) darauf hin, dass die Vorherrschaft der spirantisierten Aussprache von -g im In- und Auslaut zunehmend durch den stimmlosen Verschlusslaut abgelöst werde, und dies wohl auch durch schriftsprachlichen Einfluss (Viëtor 1901: V f.), während Siebs einige Jahrzehnte später schreibt, dass wohl vor allem die Aussprache von finalem - $g$ bei flektierten Formen (Tages) den Wandel befördert habe. (Siebs 1930: 16).
} 


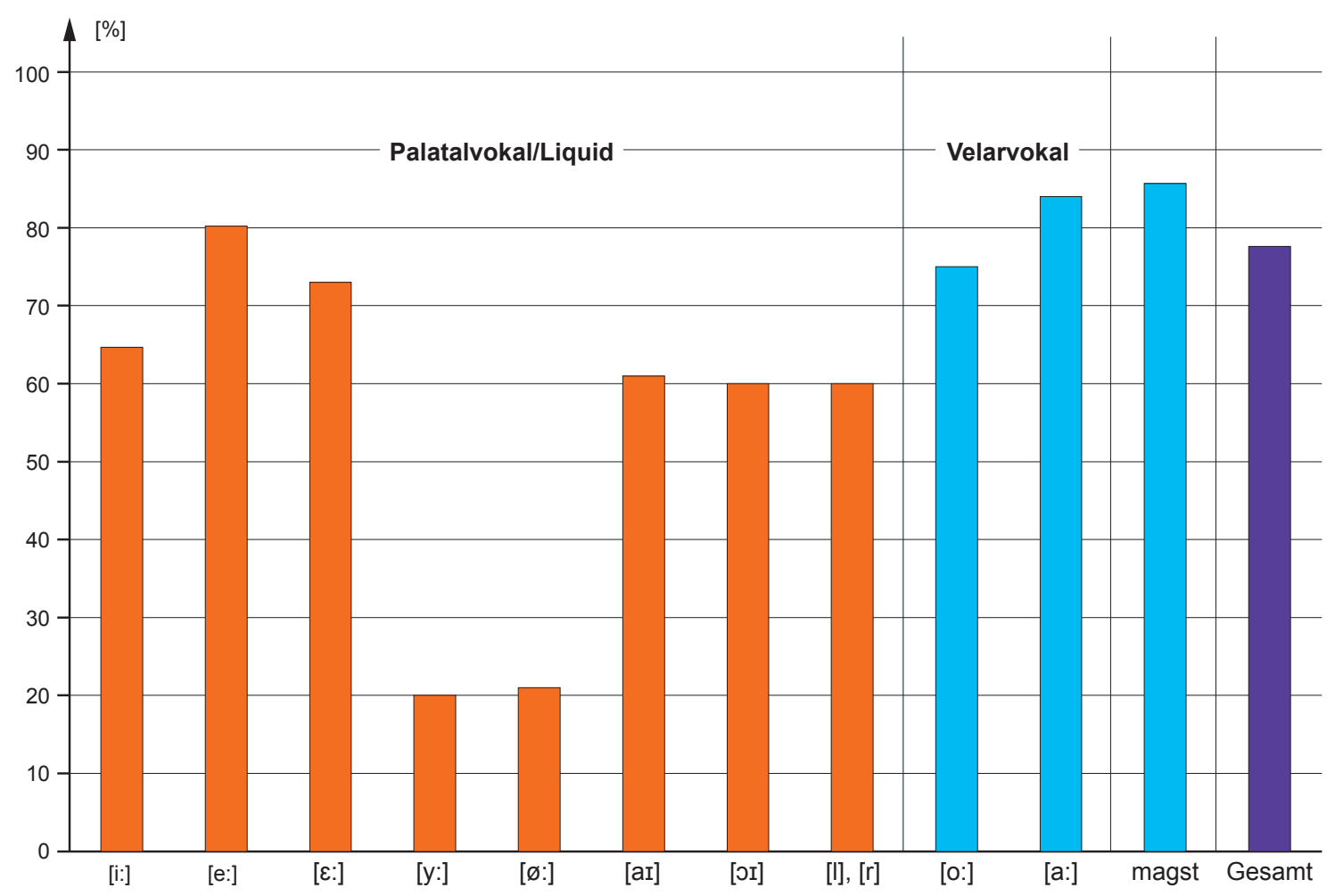

Abbildung 2: Anteil der $\boldsymbol{g}$-Spirantisierungen im Morphemauslaut

Somit kann die Variante gefracht (in Opposition zu gefragt) als eine rezente, in Norddeutschland großräumig verwendete Form und als ein ,hochfrequentes Merkmal norddeutscher Regiolekte“ (Elmentaler/Rosenberg 2015: 264) gelten. Eine daraus abgeleitete Arbeitshypothese könnte lauten, dass nur geringe regionale Unterschiede in der Spirantisierung von auslautend - $g$ wie in gefracht beobachtbar wären. Auer (1998: 189) sieht in seiner Untersuchung zur Stadtsprache Hamburgs in der finalen $g$-Spirantisierung zudem einen wenig ausgeprägten Marker für soziobiographische Gruppenzugehörigkeit (cf. auch Dahl 1974). Für den vergleichsweise geringen Anteil von Spirantisierungen beim Vorlesen verweist Auer auf den Einfluss der schriftlichen Repräsentation $<\mathrm{g}>$ für das Phonem $/ \mathrm{g} / \mathrm{im}$ Unterschied $\mathrm{zu}<\mathrm{ch}>$ für /ç/ und / $\mathrm{x} /$, was auf eine graphemische Lesweise hindeute. Ich komme darauf weiter unten zurück.

Das SiN-Korpus erlaubt es nun, zu klären, ob sich die spätestens Ende des 19. Jahrhunderts mit der Normierung der Orthoepie des Hd. forcierte Präferenz von finalem - $g$ nach Velarvokal als stimmlose velare Plosive (und der Deklassierung der Spirans als Non-Standard-Variante mit Ausnahme nach Palatalvokal wie in -ig) als schriftinduzierte und zumindest anfangs auch soziopragmatisch markierte Variante im gesamten norddeutschen Raum gleichmäßig fortund durchgesetzt hat. Oder aber, ob es regionale Unterschiede im rezenten Gebrauch dieser Variante gibt, etwa durch das Vorhandensein eines nd. Pols im Varietätenspektrum. Möglicherweise könnte die finale $g$-Spirantisierung weniger ein soziobiographisches als vielmehr ein soziopragmatisches Merkmal insofern sein, als dieses Feature entweder im Repertoire einzelner Sprecher oder im Sprachlagenspektrum einer Region eine unterschiedliche Stelle besetzt. ${ }^{4}$

\footnotetext{
${ }^{4}$ Für den Status der $g$-Spirantisierung im Ripuarischen und Thüringischen cf. Schröder 2013.
} 
Etwas verkürzt lautet die Frage: Ist die Spirantisierung von - $g$ nach Velarvokal in gedeckter Stellung wie in sagt oder gefragt in Norddeutschland überhaupt markiert, genauer: Regional und/oder soziopragmatisch markiert?

\section{$3 \quad$ Wahrnehmungsdaten und Produktionsdaten}

Eine erste Antwort geben die Salienztests zu gefracht. Diese perzeptionslinguistisch angelegten Tests bestehen aus einer Folge auditiver Stimuli in Form von kurzen Sätzen, in denen jeweils eine phonologische oder morphosyntaktische Variante realisiert wird, die vom sprechsprachlichen hd. Standard bzw. von der grammatischen Norm des Hd. abweicht. Nehmen die Gewährspersonen diese Variante als Abweichung von ihrer in der jeweiligen Testsituation aktivierten Vorstellung eines sprechsprachlichen Standards oder aus anderen Gründen als auffällig wahr, dann ist diese Form für diese GP ,salient“. Um regionale Unterschiede sichtbar zu machen, werden die Testergebnisse auf die verschiedenen Erhebungsregionen des SiNProjekts bezogen, wie sie auf Karte 1 eingezeichnet sind.

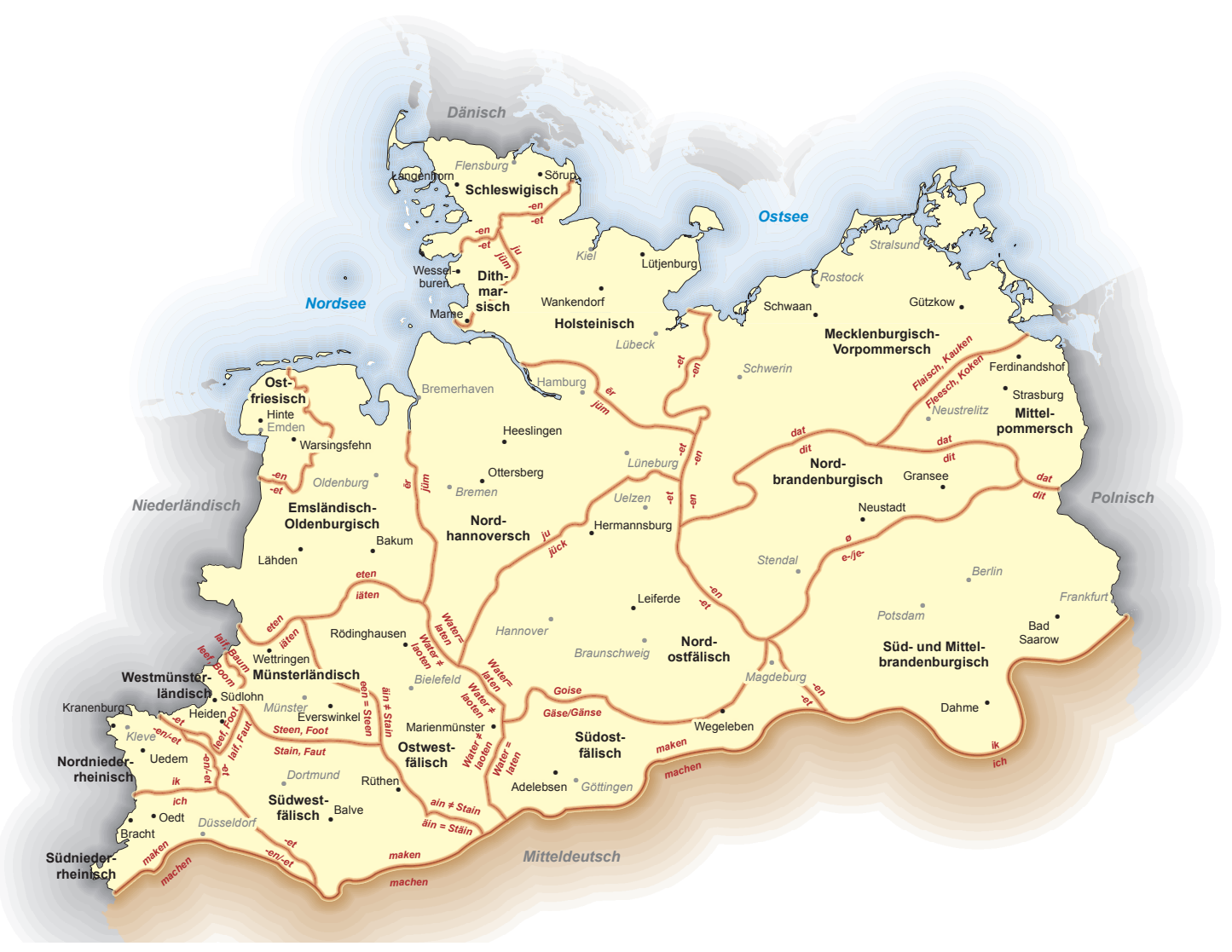

Karte 1: Erhebungsorte und -regionen

Das Diagramm (Abb. 3) zeigt eine regional unterschiedliche Verteilung der Testresultate mit einer leicht zunehmenden Tendenz des Anteils der Salienzwerte in West-Ost und Süd-NordRichtung, wenn überhaupt. ${ }^{5}$

5 Die Siglen verweisen auf die Untersuchungsregionen NN (Nordniederrheinisch), SN (Südniederrheinisch), WML (Westmünsterländisch), OFL (Ostfriesisch), EMS (Emsländisch), ODL (Oldenburgisch), ML (Münsterländisch), SW (Südwestfälisch), OW (Ostwestfälisch), SL (Schleswigisch), DT (Dithmarsisch), HO (Holstei- 


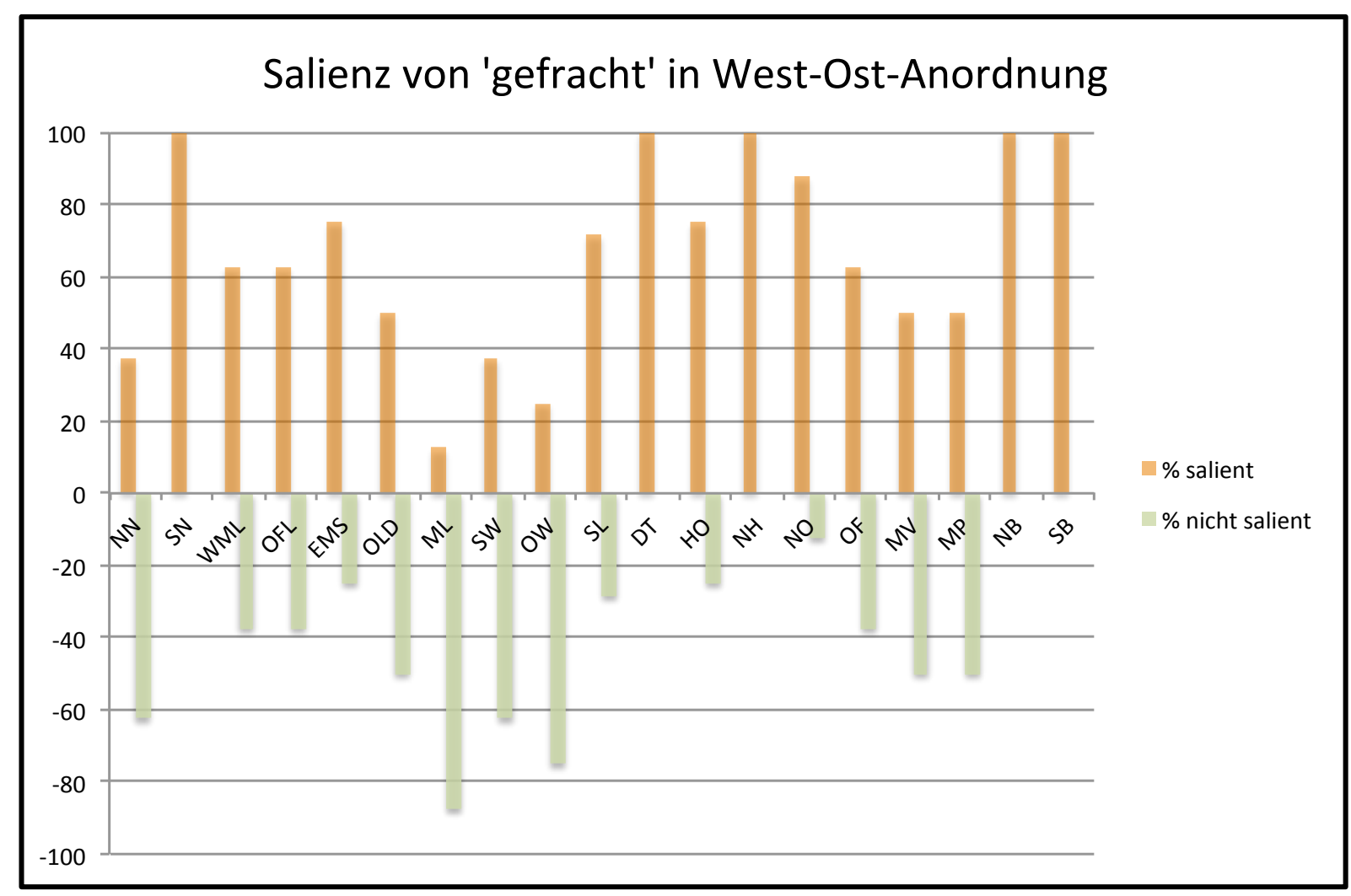

Abbildung 3: Salienz von gefracht

Aus dieser Verteilung ergeben sich zwei Fragen: Warum ist gefracht für einen Teil der GP salient, für einen anderen nicht salient - und beides in regional unterschiedlichem Umfang.

Hier einige der möglichen Hypothesen:

a. gefracht ist salient, weil gefracht im Varietätenspektrum der GP eine auffällige „Non-Standard-Variante“ zu gefragt ist (,standardsprachlicher“ Normhorizont)

b. gefracht ist salient, weil es zum sprachlichen Inventar des Ortes oder der Region (in Abgrenzung zur Standardvarietät) gehört (,,regiolektaler“ Normhorizont)

c. gefracht ist salient, weil es nicht zum „standardsprachlichen Repertoire“ der GP und zugleich als ,regiolektale“ Form klassifiziert wird (,,doppelter“ Normhorizont)

d. gefracht ist salient, weil der GP diese Form „,in der gegebenen Testsituation“ als auffällig erscheint (,Situativität“ der Stimuli, womöglich methodisches Artefakt)

e. gefracht ist salient, weil die GP diese Form aus was auch immer für Gründen nicht verwendet (,idiodsynkratischer Usus“)

In den Fällen, in denen gefracht für die GP keine Auffälligkeit darstellt, treffen die genannten Hypothesen entweder nicht zu oder die GP hat das Feature schlicht nicht wahrgenommen. Dennoch lassen sich in gewissem Umfang auch in diesen Fällen durch die Vielzahl unterschiedlicher Datentypen (Mental Maps, Kommentierungen, soziobiographische Daten, phone- 
tische Abstandswerte, Produktionsdaten) plausible Aussagen über Normhorizonte und andere soziopragmatische Faktoren treffen. Diese Hypothesenvielfalt hat dazu geführt, dass im SiNProjekt „Salienz“ als Arbeitsbegriff zunächst relativ offen bestimmt wurde: Bei Salienz sollte es sich nicht um eine statisch zu bestimmende Eigenschaft sprachlicher Formen handeln, sondern um ein ,dynamisches und relationales Konstrukt [...], das sprachliche Varianz in Beziehung zu sozial-evaluativen Instanzen setzt" (Gessinger 2008a: 67), also um eine bestimmte (Sprech)handlungen motivierende Vorstellung, in die wahrgenommene Eigenschaften der jeweiligen sprachlichen Form, Wissensbestände, Einstellungsformationen, Kontexte und Situationen interaktiv eingearbeitet werden. ${ }^{6}$ Diese Fassung des Salienzbegriffes, zu dessen Präzisierung und Operationalisierung Purschke (2011) mit der Differenzierung von Salienz, Salienzpotential und Pertinenz und einer im wesentlichen handlungstheoretisch fundierten Zuordnung von Einstellung, Evaluation und der Aktivierung von Wissensbeständen (Purschke 2015) einen wichtigen Beitrag geleistet hat, ${ }^{7}$ berïcksichtigt insbesondere die methodischen Implikationen, die aus dem spezifischen Kontext der Datenerhebung resultieren, hier vor allem der Einfluss der Interaktion zwischen Gewährspersonen und Exploratoren auf die Wahrnehmung und Beurteilung sprachlicher Varianz (cf. Gessinger/Butterworth 2015).

Um die Mehrdeutigkeit von Salienz zu reduzieren, folgen im Testdesign des SiN-Projekts auf den Salienztest ein „Situativtest“ und ein „Normativitätstest“, in denen die als salient wahrgenommenen Merkmale in teilweise anderer sprachlicher Umgebung auf ihre mögliche Verwendung in unterschiedlich formalisierten situativen Kontexten abgefragt und anschließend auf ihre Akzeptabilität bei der Verwendung durch andere in einem fiktiven halbformellen Kontext geprüft werden.

Zunächst aber noch ein zweiter Blick auf die Produktionsdaten (Elmentaler/Rosenberg 2015: 262):

\footnotetext{
${ }^{6}$ Cf. Garret/Coupland/Williams 2003.

7 Cf. dazu exemplarisch Lenz 2010, Schmidt/Herrgen 2011, Kiesewalter 2011, Rácz 2013, Schröder/Palliwoda 2016 und die Beiträge in Linguistik online 66,4 (2014) sowie aus dem Kontext des SiN-Projekts Gessinger 2008a und 2008b, Elmentaler/Gessinger/Wirrer 2010, Butterworth/Glawe 2011 und Hettler 2013.
} 


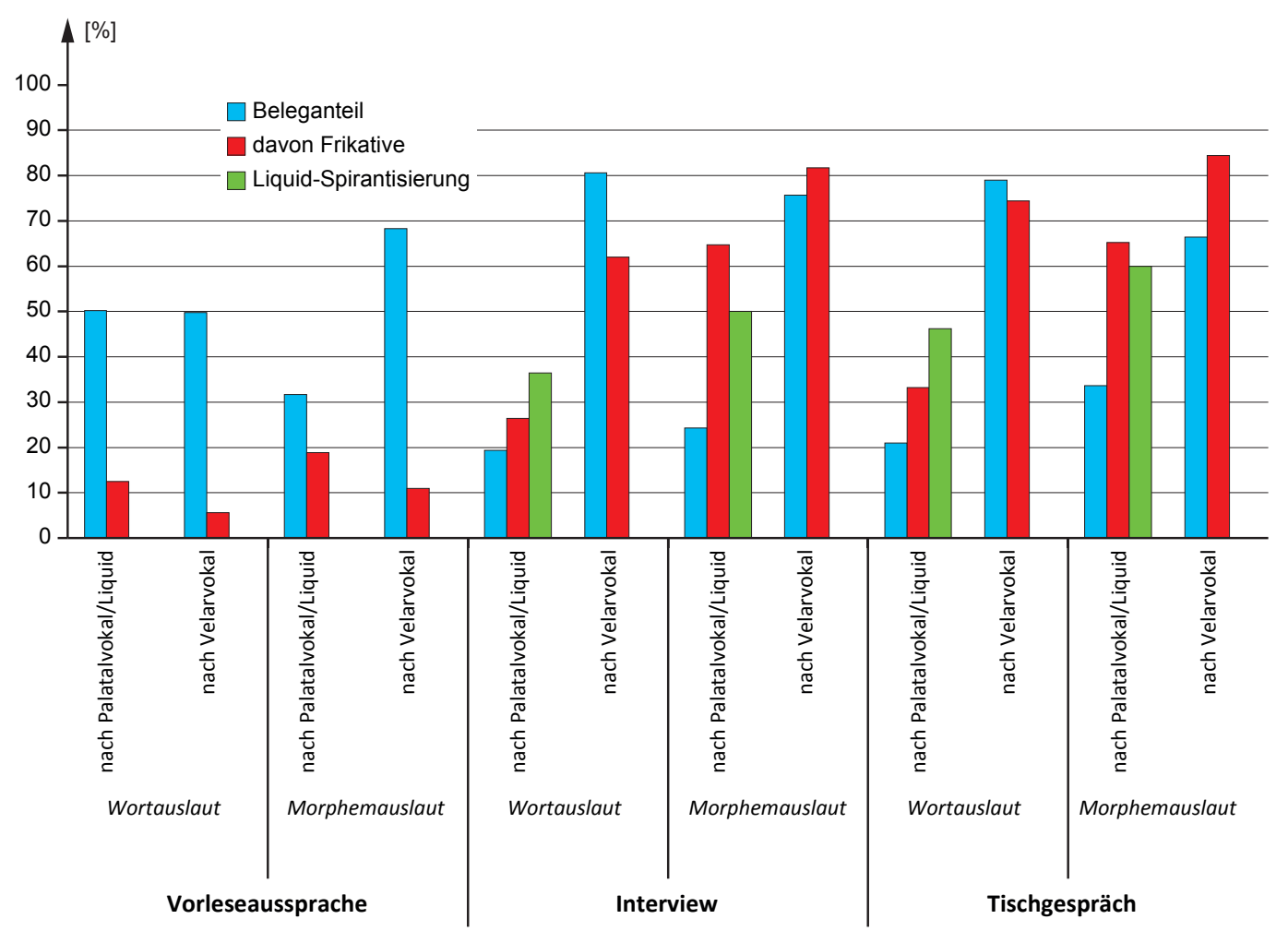

Abbildung 4: Produktionsdaten zur g-Spirantisierung im Auslaut

Dieses Diagramm aus dem Atlasband zeigt, dass der Anteil der Realisierungen von gSpirantisierungen an allen Vorkommen von $-g$ im absoluten Wort- und Morphemauslaut in unterschiedlich formalisierten Kontexten unterschiedlich hoch ist. Während in der Vorleseaussprache (intendiertes Hd.) der Anteil von $g$-Spirantisierungen im Morphemauslaut nach Velarvokal wie in gefracht bei $11 \%$ liegt, sind es in den Interviews und in den informellen Tischgesprächen über $80 \%$. Diese Ergebnisse zeigen, dass die finale $g$-Spirantisierung im intendierten Hd. nur in geringem Umfang realisiert wird. In den halb- oder informellen Kontexten (Interview und Tischgespräch) ist diese Variante hingegen hochfrequent.

Mit anderen Worten: Die situative Differenz zwischen „formellem“ und „nicht-formellem“ Kommunikationskontext scheint für die meisten GP den Normhorizont für die Verwendung der spirantisierten Variante zu bestimmen.

Als erstes Zwischenergebnis lässt sich festhalten, dass die Salienz von gefracht unterschiedlich ausgeprägt ist, ebenso die situative Verteilung der $g$-Spirantisierung im Morphemauslaut in den Produktionsdaten. Beide Parameter zeigen zudem regionale Unterschiede, auf die ich weiter unten eingehen werde.

Für einen Salienzbegriff, wie er etwa noch von Trudgill (1986) vertreten wurde, ist die Hypothese konstitutiv, dass Sprecher saliente Formen zunehmend vermeiden würden, je stärker die Situation formalisiert sei. Chambers (2009) verweist auf die Beobachtung, dass ein formelleres Gespräch mit ausgeprägterem Korrekturverhalten von Non-Standard-Formen und verstärktem Self-monitoring der Sprecher einhergehe.

Wenn dies zuträfe, dann müsste es einen Zusammenhang zwischen Salienz und der Realisierung der $g$-Spirantisierung in den Produktionsdaten geben, die ja bezogen auf drei Kontexte 
mit unterschiedlichem Formalitätsgrad erhoben wurden: Tischgespräch in Abwesenheit der Exploratoren, Interview und Vorleseaussprache (intendiertes Hd.) mit zunehmendem Formalitätsgrad. Die Abbildung 5 zeigt die Ergebnisse des Salienz-Tests für gefracht $(0=$ nicht salient, 1 = salient, bezogen auf 142 von insgesamt 144 Gewährspersonen), Abbildung 6 die Anteile der $g$-Spirantisierungen bei den Produktionsdaten in den drei unterschiedlichen Erhebungskontexten.
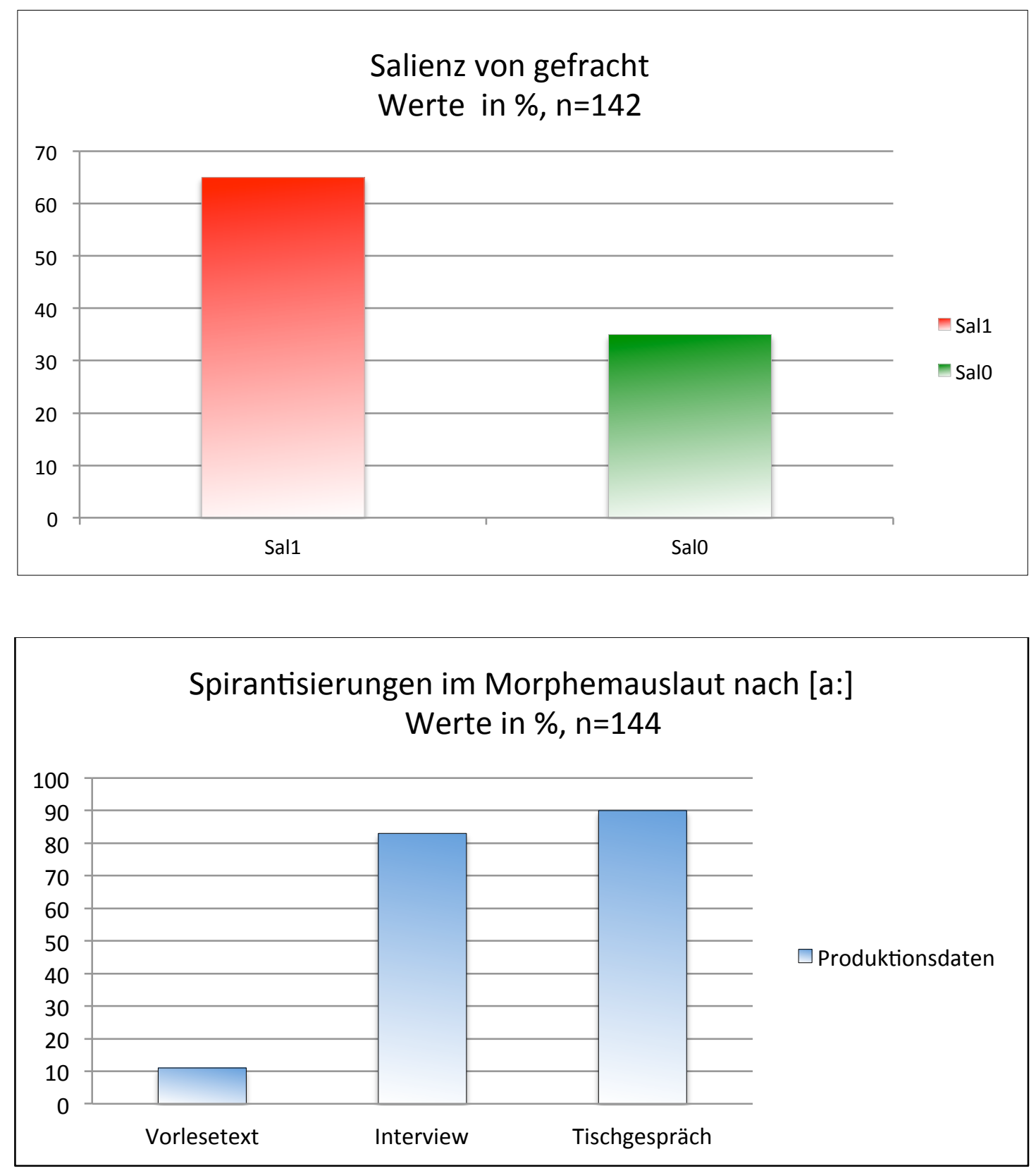

Abbildung 5, 6: Salienz und Produktionsdaten in drei Kontexten mit unterschiedlichem Formalitätsgrad

Die graphische Darstellung der Abhängigkeit von Salienz und $g$-Spirantisierung in der Vorleseaussprache zeigt folgendes Bild: 


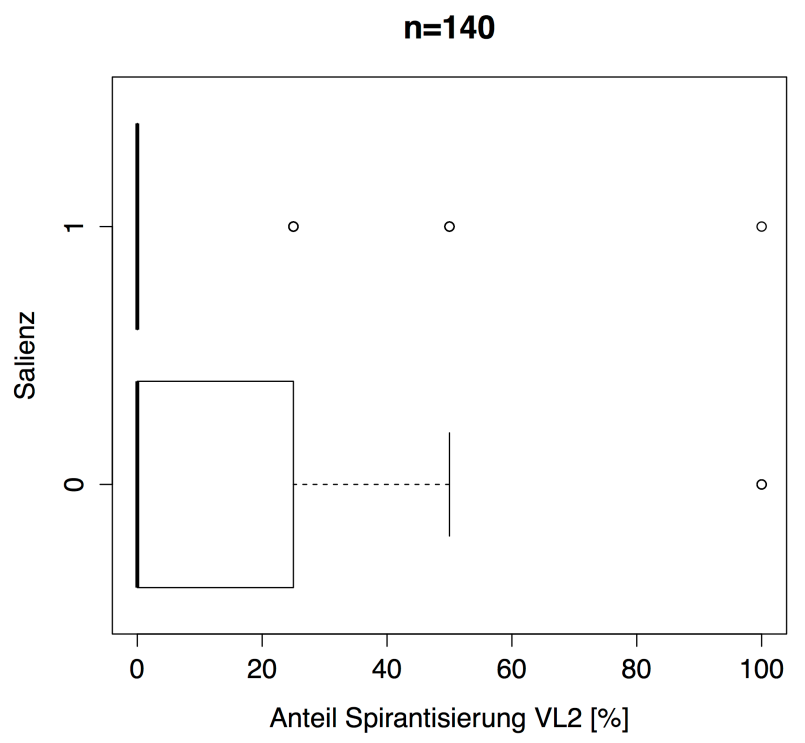

Abbildung 7: Boxplot Salienz Vorleseaussprache

Zwar ist erkennbar, dass für die Gruppe der GP, für die gefracht nicht salient ist, der Wert stärker streut, aber richtig interpretierbar ist der boxplot nicht, weil die große Mehrheit der GP in der Vorleseaussprache nicht spirantisiert. Die postulierte Abhängigkeit von Salienz und Frequenz der Spirantisierungen in der Vorleseaussprache ist statistisch nicht signifikant.

Gibt es nun vielleicht einen Zusammenhang zwischen Salienz und den Spirantisierungen in den weniger formellen Situationen, im Interview und im Tischgespräch?
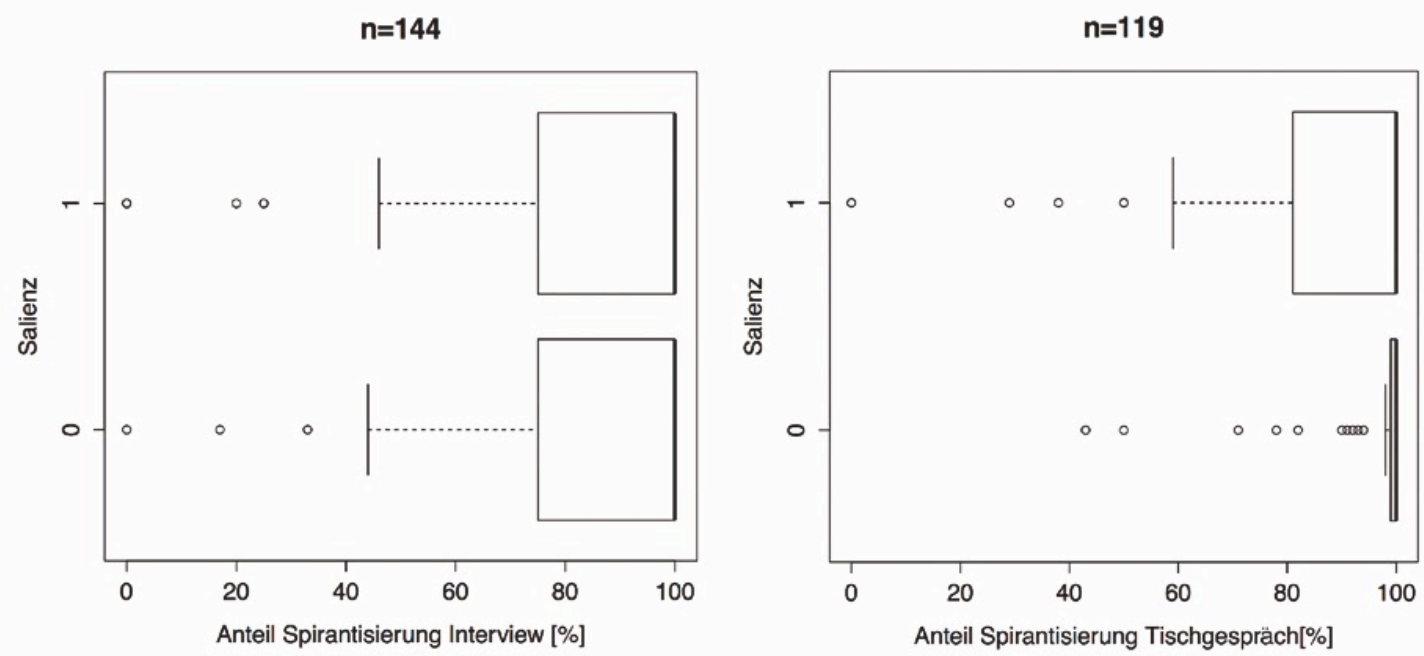

Abbildung 8, 9: Boxplots Salienz Interview und Salienz Tischgespräch

Während für das Interview der Faktor Salienz erkennbar keinen Einfluss hat, weisen die Daten aus dem Tischgespräch zwar eine etwas größere Streuung für vorhandene Salienz auf, in beiden Fällen aber liegt die größte Häufigkeit der Spirantisierungen nahe 100\%, der Unterschied in der Häufigkeitsverteilung ist nicht signifikant (Das Sample ist im Tischgespräch kleiner, weil dort $25 \%$ der Gespräche niederdeutsch geführt wurden).

Als zweites Zwischenergebnis lässt sich festhalten, dass sich ein Einfluss des Faktors Salienz auf die Häufigkeit von Spirantisierungen in den Produktionsdaten statistisch nicht belegen 
lässt. ${ }^{8}$ Die Hypothese, die eine stärkere Vermeidung salienter Formen in zunehmend formellen Situationen erwartet, wird nicht bestätigt. Festzuhalten bleibt aber der manifeste Unterschied in der Gebrauchsfrequenz der finalen $g$-Spirantisierung nach Velarvokal [a:] zwischen Vorleseaussprache, Tischgespräch und Interview (Abb. 6).

Wenn für die meisten GP nicht die Salienz einer Variante, sondern der Formalitätsgrad einer Situation die Verwendung der spirantisierten Variante steuert, dann bietet es sich an, den Faktor „Situativität“ für die Produktionsdaten mit den Resultaten des Situativitätstests aus der Testbatterie in Beziehung zu setzen. Dabei gehe ich von der Annahme aus, dass sich die implikationsbasierte Skalierung im Situativitätstest (Variante wird nie, nur informell, auch in halbformellen Situationen, auch in formellen Situationen, d. h. in allen Situationen gebraucht) mit den entsprechenden Formalitätsgraden der Produktionsdaten vergleichen lässt, wie es das folgende Schema zeigt:

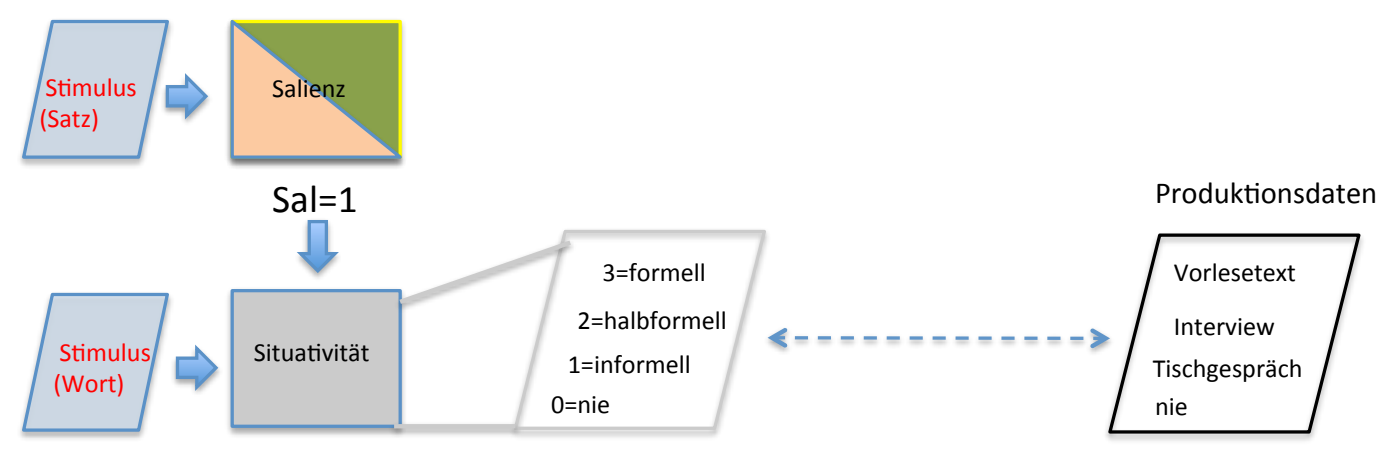

Abbildung 10: Beziehung zwischen Situativitätstest und Produktionsdaten

Wie schon in Abb. 6 erkennbar, zeigt die genauere Aufschlüsselung der Produktionsdaten für die drei Situationen mit unterschiedlichem Formalitätsgrad deutliche Differenzen bei den jeweiligen Anteilen der finalen $g$-Spirantisierungen nach Velarvokal [a:]:

\footnotetext{
${ }^{8}$ Gleiches gilt auch für den Parameter „Normativität“, der gleichfalls nicht mit den Produktionsdaten korreliert.
} 


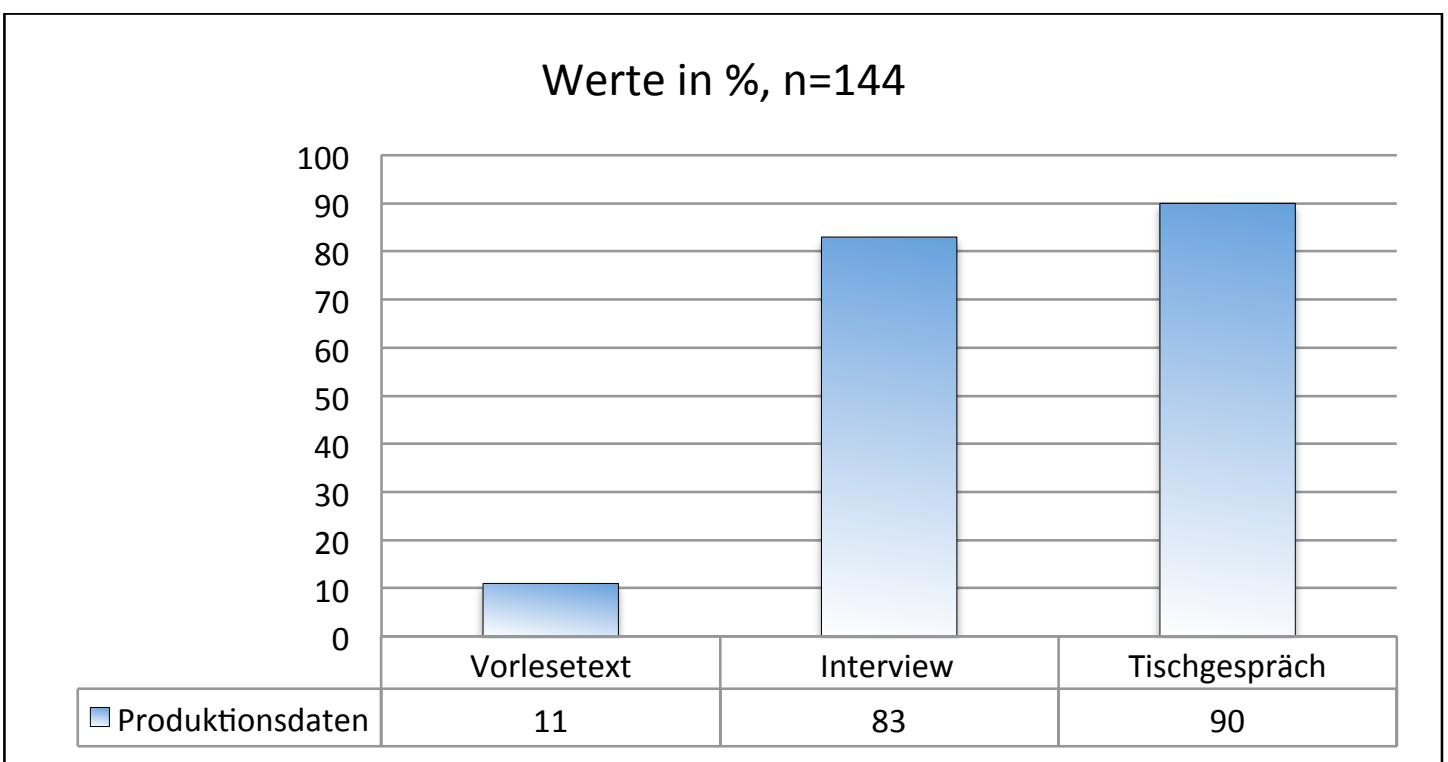

Abbildung 11: g-Spirantisierung nach Velarvokal [a:] in Situationen mit unterschiedlichem Formalitätsgrad

Da die Salienz als „Filter“ für die folgenden Situativitäts- und Normativitätstests verwendet wurde $(\mathrm{SAL}=1)$, gelten die folgenden Werte nur für diejenigen GP, für die gefracht salient war, d. h. das Sample ( $\mathrm{n}=93)$ ist kleiner.

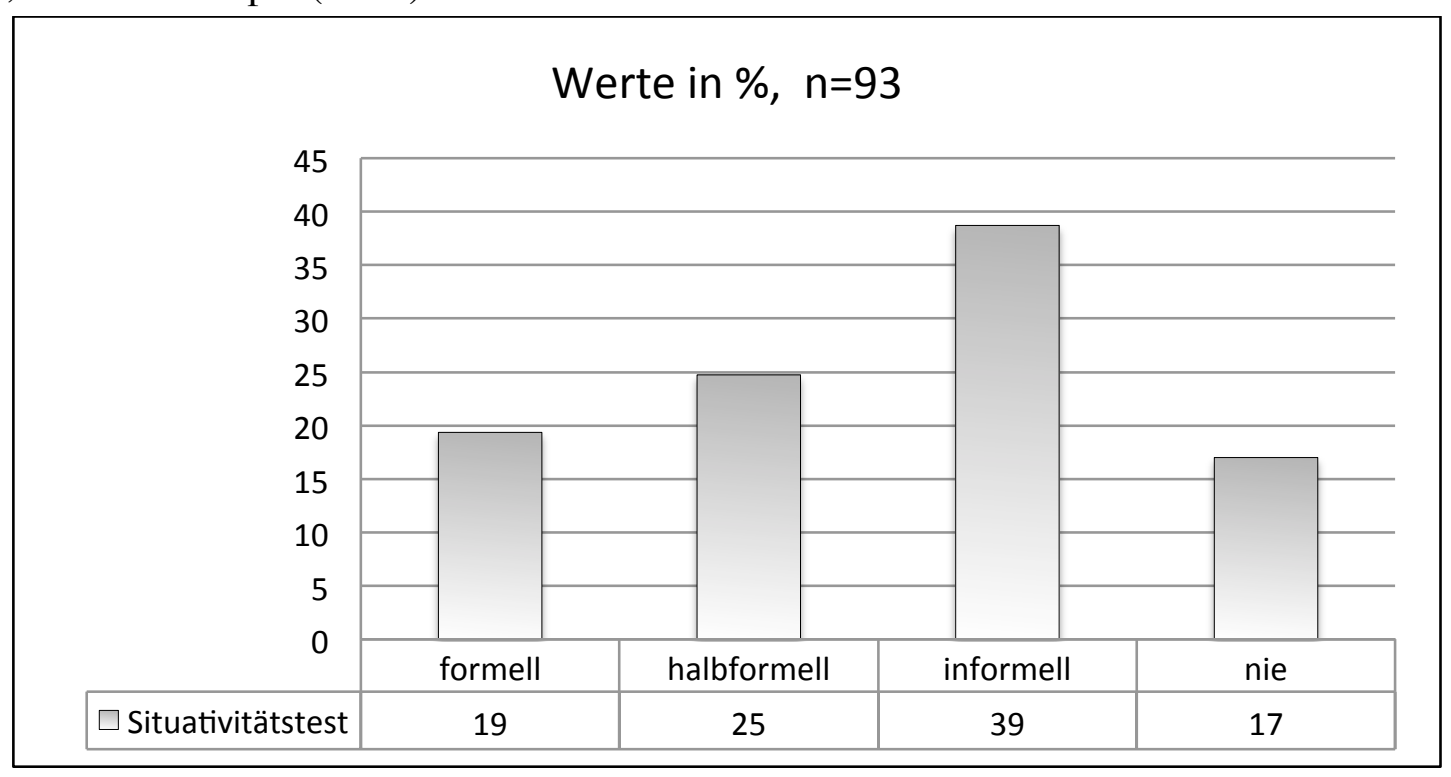

Abbildung 12: Situativität von gefracht

Die Mehrzahl der GP (39\%) gibt an, gefracht nur in informellen Situationen zu verwenden, $25 \%$ in halbformellen und $19 \%$ sogar in formellen Kontexten. $17 \%$ der GP würden diese Variante überhaupt nicht verwenden.

Die Frequenz der Realisierungen der $g$-Spirantisierung zeigt, dass der Normhorizont nicht nur bei den Testdaten, sondern auch beim Gebrauch dieser Variable in der situativen Differenz zwischen formellen Kontexten mit standardsprachlicher Orientierung und nicht-formellen Kontexten mit Verwendung der Non-Standard-Variante liegt. Diese Interpretation wird dadurch gestützt, dass die Werte für Interview und Tischgespräch sich erstaunlich wenig unterscheiden. Außerdem wird hier auch deutlich, dass der Einfluss der Graphie <ch $>$ vs. $<$ g $>$ 
bei den Perzeptionstests jedenfalls nicht die entscheidende Rolle spielen kann, denn der Stimulus für gefracht wurde auditiv gesetzt.

Ein Boxplot macht das Verhältnis der Wahrnehmungsdaten (Situativitätstest) zu den Produktionsdaten (Spirantisierung im Interview) anschaulich:

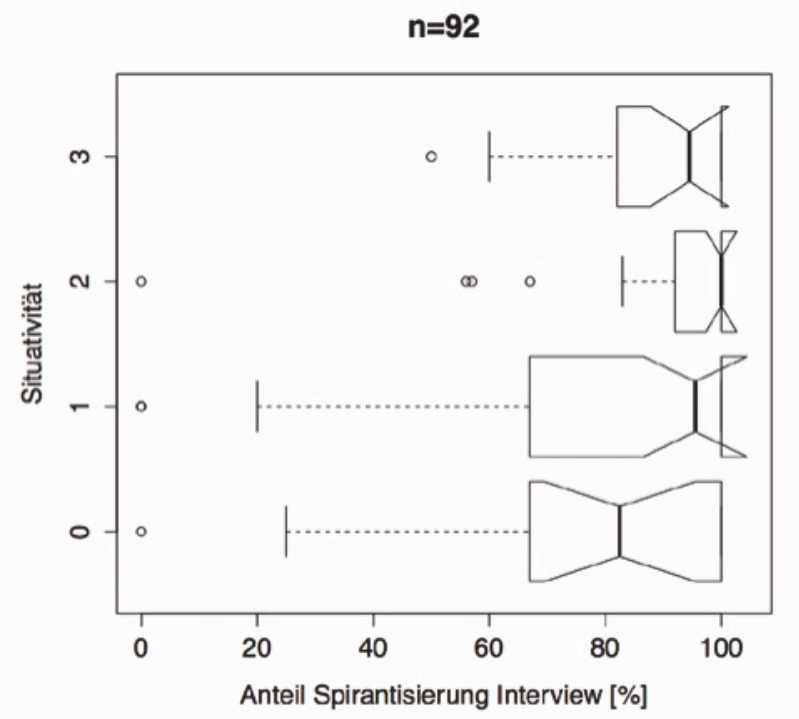

Abbildung 13: Häufigkeitsverteilung der Spirantisierungen im Interview in Abhängigkeit von Situativität

Hier zeigt sich, dass der Anteil der Spirantisierungen im Interview größer wird, je eher die GP diese Variante auch in formellen Situationen zu verwenden bereit waren. ${ }^{9}$

Mit einiger Sicherheit lassen diese Daten darauf schließen, dass die Wahl in der Verwendung der spirantisierten und nicht-spirantisierten Variante nicht von der Salienz, sondern vom Formalitätsgrad des situativen Kontextes beeinflusst wird, der in der Beurteilung der eigenen Sprachpraxis, wie die Wahrnehmungsdaten zeigen, zur normativ wirksamen Instanz wird.

\section{Produktionsdaten, Wahrnehmungsdaten, Arealität}

Die bisher gebrauchte Unterscheidung von Standard und Non-Standardvarianten lässt sich allerdings noch präzisieren, wenn die im SiN-Atlasband kartierte areale Differenzierung in der Häufigkeit situativ unterschiedlicher Verwendungen genauer betrachtet wird:

\footnotetext{
${ }^{9}$ Zur Erläuterung des Boxplots: Die Skala auf der Ordinatenachse zeigt die vier möglichen Angaben im Situativitätstest, d. h. die GP gibt an, gefracht nie (0), nur informell (1), auch halbformell (2), immer (3) zu verwenden. Der Median, der anzeigt, wo der Anteil von 50\% der Belege in dem von der Box dargestellten Wertebereich von $25 \%-75 \%$ liegt, rutscht immer weiter nach rechts gegen $100 \%$, d.h. im Interview wird die finale $g$-Spirans mehrheitlich bis ausschließlich verwendet. Zugleich nimmt die Streuung von $0 \quad 2$ ab. Nur der Unterschied zwischen den Häufigkeitsverteilungen und Frequenzen der Gruppe 0 (unterster plot, „,verwende die Variante nie“) und Gruppe 2 (,verwende sie auch in halbformellen Situationen“) ist statistisch signifikant, wie die „notches" zeigen.
} 


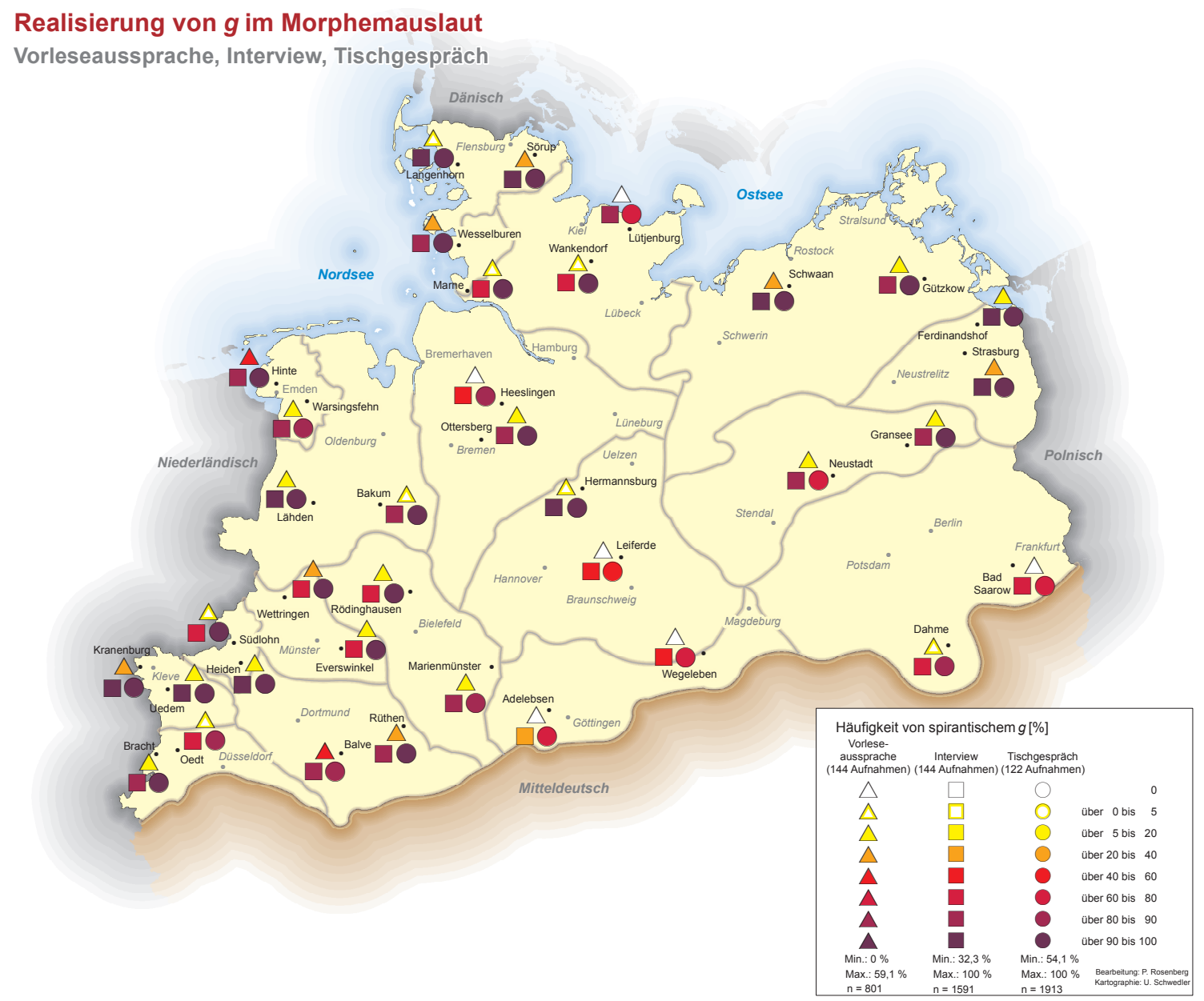

Karte 2: Regionale Verteilung der Produktionsdaten für $g$-Spirantisierungen im Morphemauslaut

Im Vergleich der verschiedenen situativen Kontexte zeigt sich eine deutliche Abstufung zwischen Interview (Quadrate) und Tischgespräch (Kreise) gegenüber der Vorleseaussprache (Dreiecke), in allen Kontexten wird zudem in regional unterschiedlichem Maße spirantisiert (die dunkleren Farben indizieren jeweils eine höhere Gebrauchsfrequenz der $g$-Spirans).

Um diesen Befund etwas detaillierter darstellen zu können, habe ich sechs Erhebungsregionen ausgewählt, die sich in den Produktionsdaten deutlich unterscheiden. 


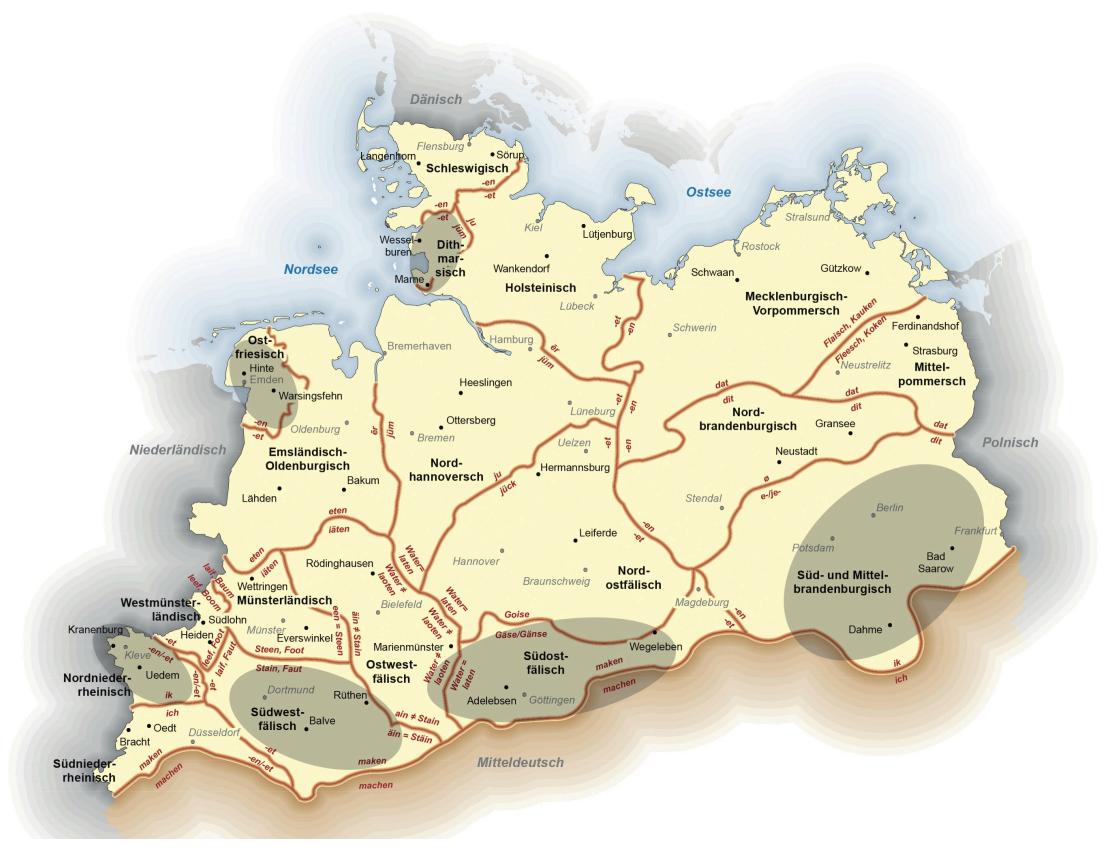

Karte 3: Ausgewählte Erhebungsregionen ${ }^{10}$ für gefracht.

Die folgende Übersicht zeigt den prozentualen Anteil der finalen $g$-Spirantierungen für die Produktionsdaten, aufgeschlüsselt nach sechs Erhebungsregionen und -orten sowie situativen Kontexten.
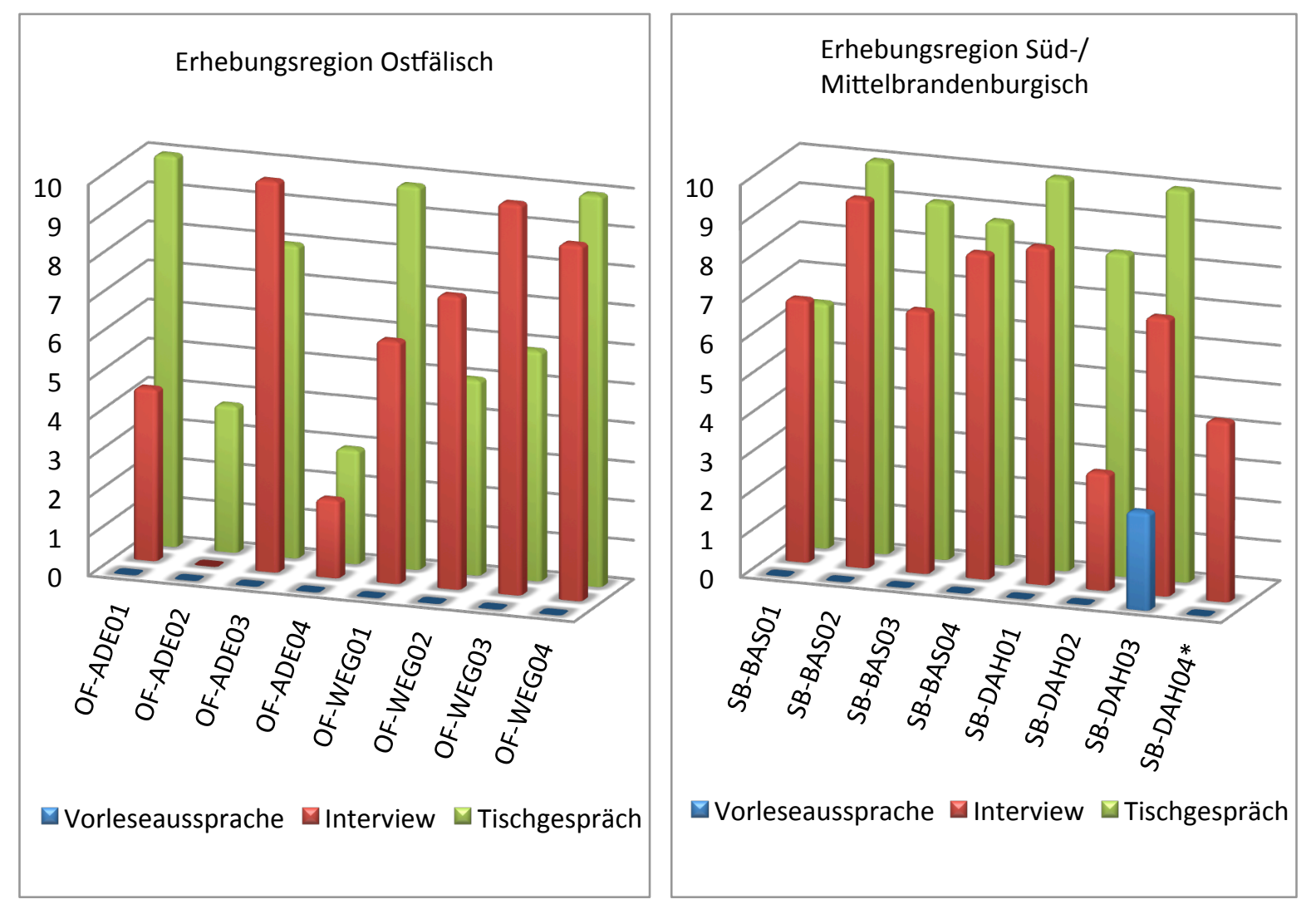

10 Ausgewählte Erhebungsregionen und -orte (hervorgehoben): OF (Ostfälisch: Wegeleben, Adelebsen), SB (Süd-/Mittelbrandenburgisch: Bad Saarow, Dahme), DT (Dithmarsisch: Wesselburen, Marne), NN (Nordniederrheinisch: Kranenburg, Uede), OFL (Ostfriesisch: Hinte, Wahrsingfehn) SW (Südwestfälisch: Balve, Rüthen). 

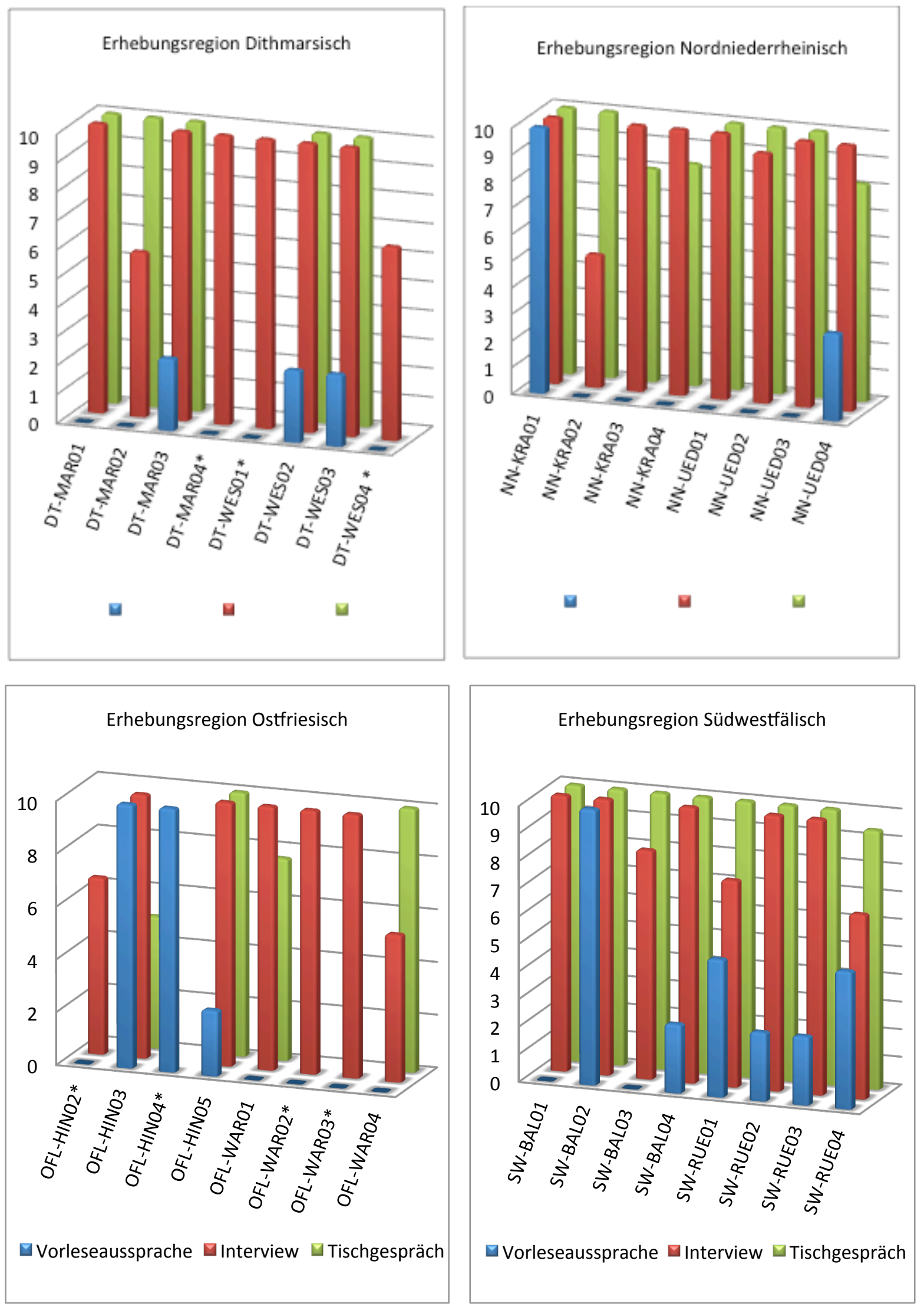

Abbildung 14: Regionale Verteilung der Spirantisierungen in drei Erhebungskontexten ${ }^{11}$

\footnotetext{
${ }^{11}$ Zur besseren Lesbarkeit wurden die Werte um dem Faktor 10 skaliert, d. h. beim Wert 10 wurden von der GP $100 \%$ der Belege für finales - $g$ spirantisiert., so beispielsweise die GP02 aus dem mittelbrandenburgischen Bad
} 
Während der Anteil der Spirantisierungen im Tischgespräch (grün) und im Interview (rot) allgemein hoch ist, wenn auch mit Differenzen vor allem in der Streuung, liegen die markanten Unterschiede bei der Spirantisierung in der Vorleseaussprache (blau). Dort liegt der Anteil für das Gesamtsample bei 11\%. Die regionale Differenzierung zeigt nun aber, dass die GP aus dem Ostfälischen (OF) und Süd- und Mittelbrandenburgischen (SB) in der Sprachlage ,intendiertes Hochdeutsch" so gut wie nie spirantisieren. Für die GP aus den Regionen Nordniederrheinisch (NN), Dithmarsisch (DT) und Ostfriesisch (OFL) ist das Bild uneinheitlich. Von den GP aus dem Südwestfälischen (SW) spirantisieren deutlich mehr auch in der Vorleseaussprache als in den anderen hier ausgewerteten Regionen. Liegt der Anteil der Spirantisierungen an den Belegen in der Vorleseaussprache bei 100\%, verfügen diese GP anscheinend nur über eine, nämlich die spirantisierte Variante, was sich auch daran zeigt, dass in diesen Fällen der Beleganteil für alle drei Kontexte (Tischgespräch, Interview, Vorleseaussprache) bei $100 \%$ liegt und so die Unterscheidung Standard vs. Non-Standard obsolet zu werden scheint, wie etwa die GP aus Kranenburg (NN-KRA01) und Balwe (SW-BAL02).

Ob es hier angemessener wäre, eher die Unterscheidung Standard vs. Regiolekt anzusetzen, lässt sich nun anhand der Mental Maps überprüfen, die von den GP aus diesen Erhebungsregionen gezeichnet wurden und eine Visualisierung derjenigen sozial- und wahrnehmungsräumlichen Konstrukte ermöglichen, die von den sprachlichen Stimuli angestoßen wurden. In diesem Fall bestand der Stimulus aus Einzelwörtern mit unterschiedlichen Non-StandardMerkmalen. Zur weiteren Auswertung wurden die so entstandenen Zeichnungen als Polygone digitalisiert und anschließend georeferenziert.

\section{$5 \quad$ Mental Maps und Normhorizonte}
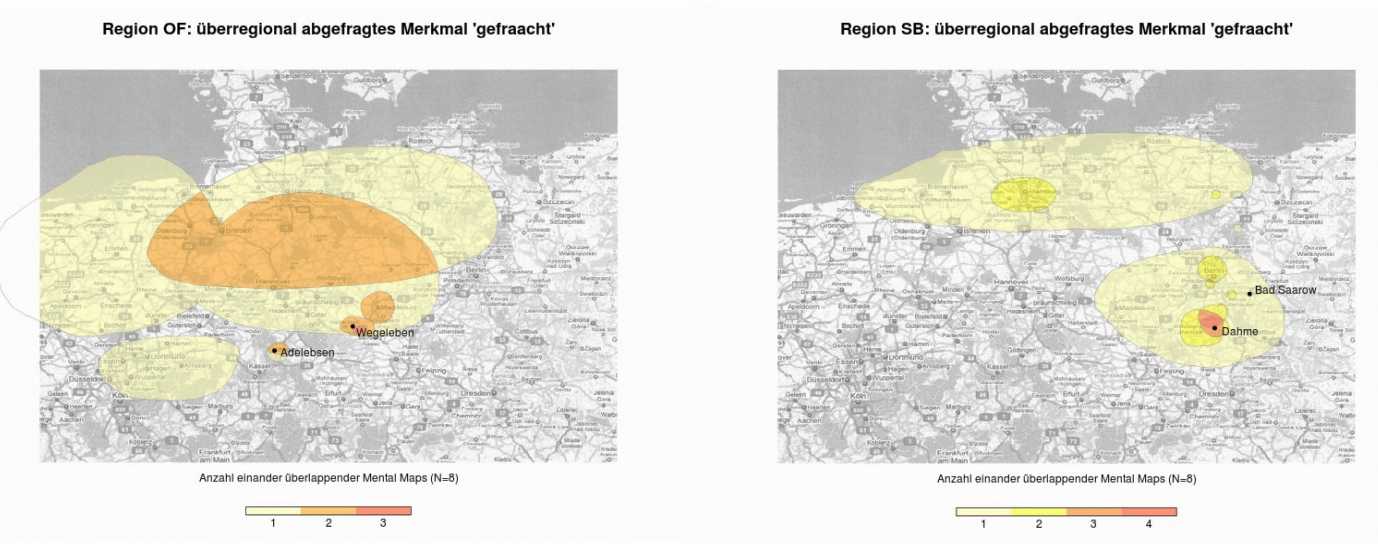

Saarow (SB-BAS02), die im Tischgespräch das finale - $g$ nach [a:] durchweg, im Interview um $90 \%$ und in der Vorleseaussprache (intendiertes Hd.) überhaupt nicht spirantisiert. Die mit Asterisk* markierten GP haben das Tischgespräch auf Nd. geführt, deshalb erscheinen hier für diese hochdeutsche Variable (grüne Balken) keine Werte. 

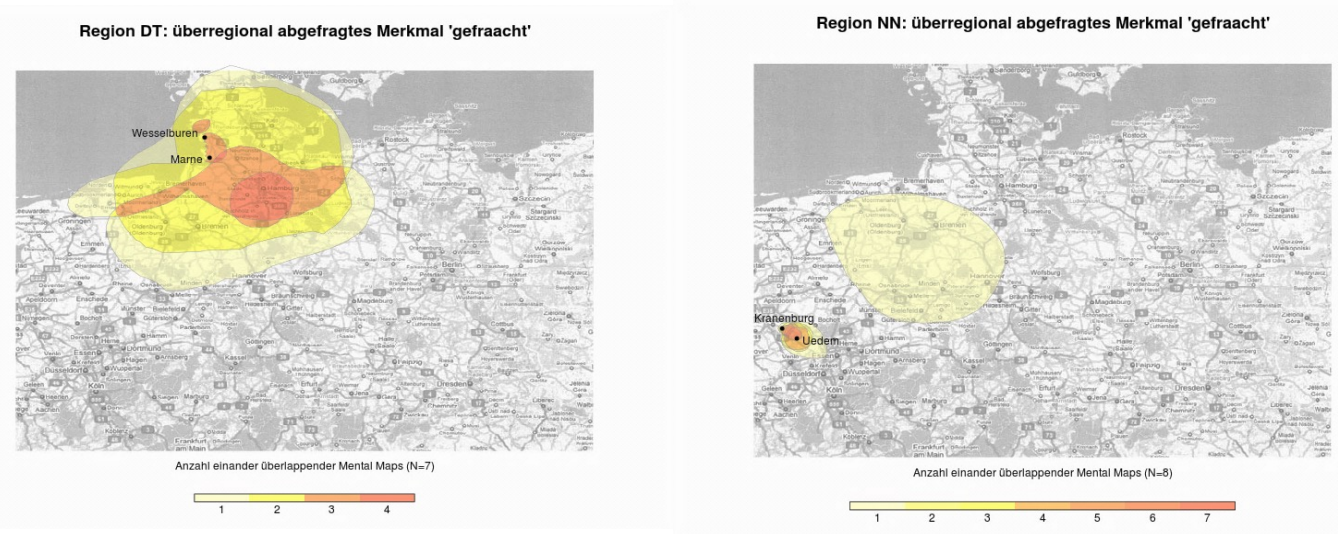

Region OFL: überregional abgefragtes Merkmal 'gefraacht'

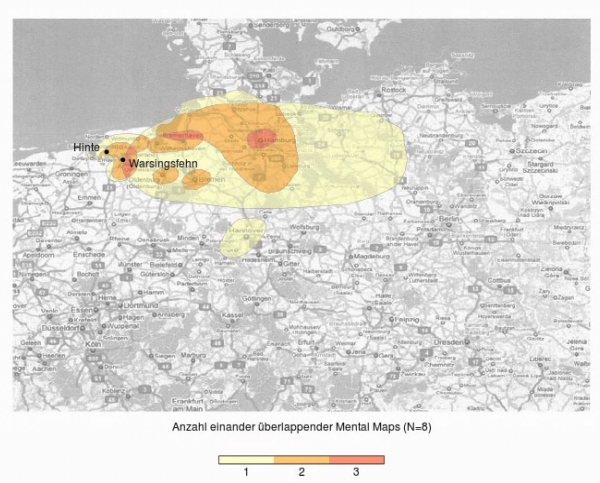

Region SW: überregional abgefragtes Merkmal 'gefraacht'

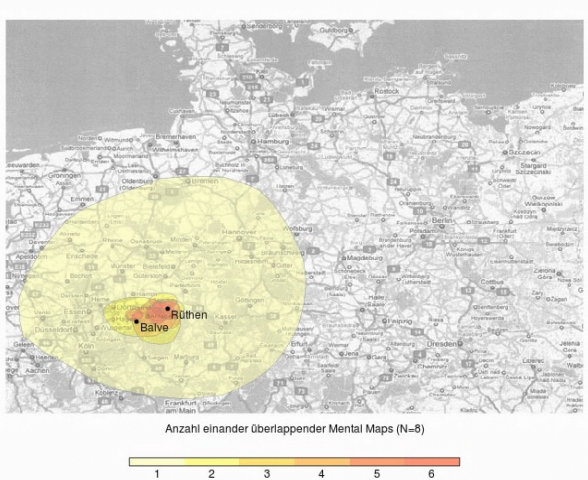

Karten 4-9: Mental Maps zu gefracht aus 6 Erhebungsregionen

Die Färbung der Polygone zeigt den jeweiligen Grad der Überdeckung in absoluten Zahlen an. Die Karten lassen drei Dimensionen erkennen: die Größe des von den GP als Verbreitungsgebiet für gefracht gezeichneten Areals, seine Form und die Lage zum Erhebungsort, dem eigenen Wohnort. Der Parameter „Lage“ indiziert, ob der Gebrauch dieser Form im unmittelbaren Erfahrungsraum ${ }^{12}$ („hier spricht man (auch) so“) oder aber in anderen, entfernten Räumen verortet wird, aus der Klein- oder Großräumigkeit in Verbindung mit der Form des Polygons und der Relation zu Orientierungsmarken auf der vorgegebenen Grundkarte ergeben sich möglicherweise auch Hinweise auf die Präzision oder Vagheit dieser Annahmen, also allgemein auf den Charakter der hier aktualisierten Wissensbestände. Vielfach unterstützen Kommentierungen beim Zeichnen die Interpretation. Vergleichsweise eindeutig (und deshalb wird dieser Parameter auch hier herangezogen) ist die Lage des Polygons: Liegt der eigene Wohnort innerhalb des eingezeichneten Verbreitungsgebiets, sprechen wir von ,autochthonen“ Kartierungen, ansonsten von ,allochthonen“ Kartierungen (cf. SW und OF). Manchmal zeichnen die GP mehrere, diskrete Verbreitungsareale, dann sprechen wir von diskontinuierlichen Kartierungen (OFL). Schließlich bleibt noch das Flächenmaß der Kartierungen, das wir anhand der Georeferenzierung berechnet haben, aber hier reicht schon der visuelle Eindruck, um Unterschiede zwischen SB, SW und OFL zu erkennen.

$12 \mathrm{Zu}$ den unterschiedlichen Raumkonzepten cf. die klassischen Arbeiten von Mannheim (1980), Cassirer (1990), Ströker (1965) und Gosztonyi (1976), zur Konstruktion und methodisch geleiteten Rekonstruktion von Sprachhandlungsräumen Lameli (2015) sowie den Literaturbericht von Paasi (2003) zum Verhältnis von Identität und Region aus humangeographischer Perspektive. 
Die Kombination aus den Produktionsdaten und der jeweiligen Lage des kartierten Verbreitungsgebiets zum Erhebungsort (violette Balken = autochthone Kartierungen) ergibt folgendes Bild:
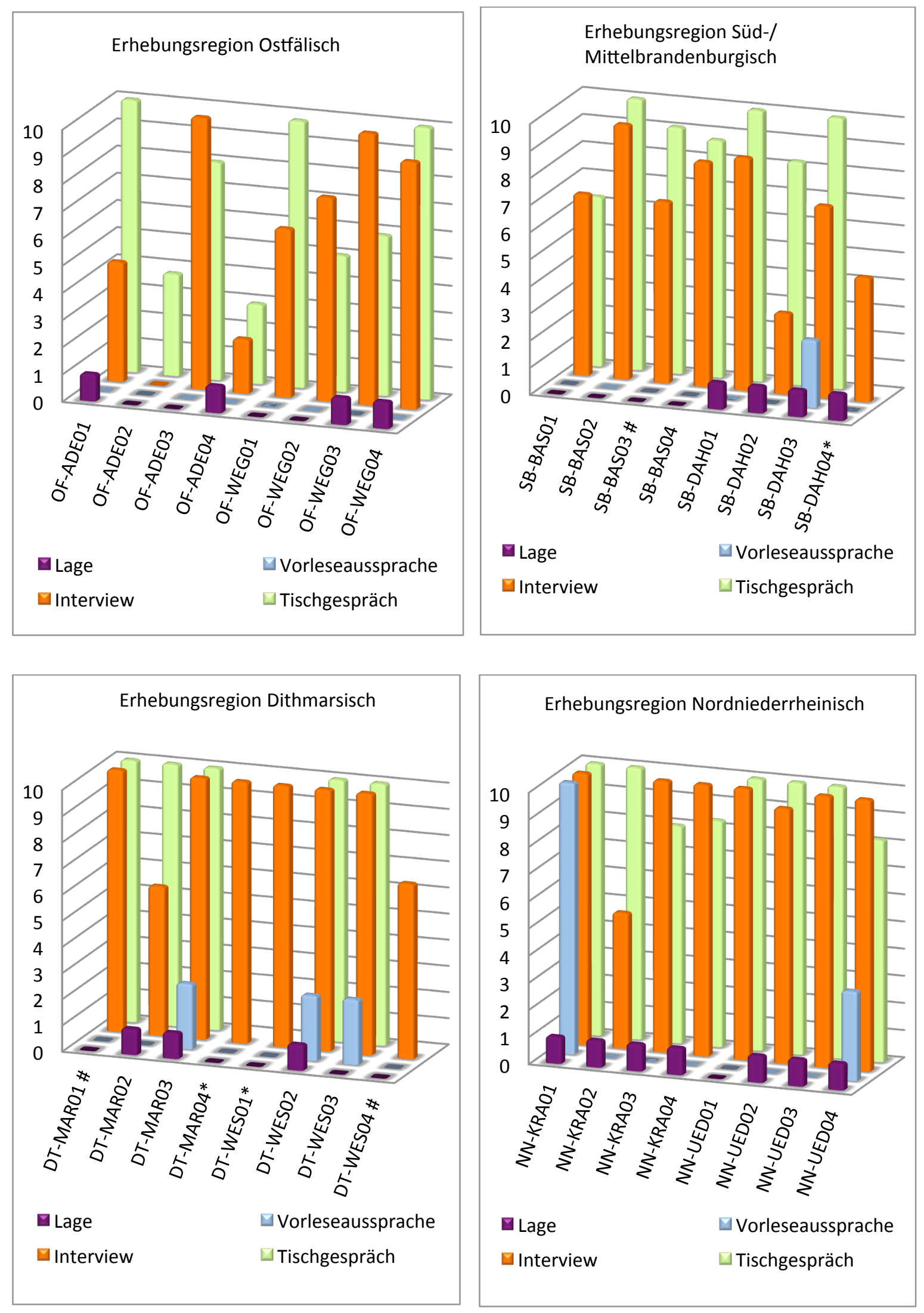

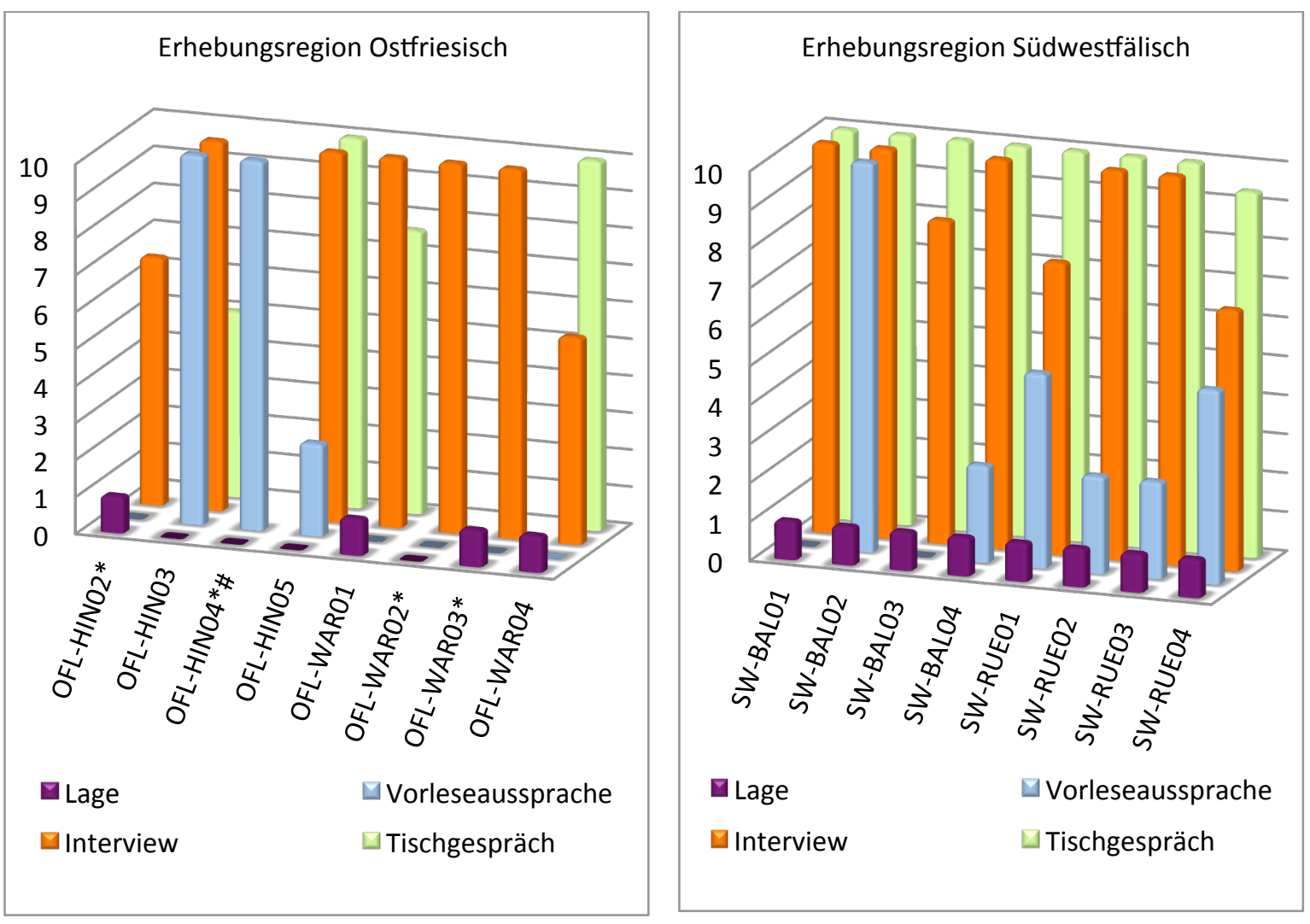

Abbildung 15: Regionale Verteilung der Spirantisierungen und Autochthonizität der Mental Maps ${ }^{13}$

Aus der bisherigen Analyse der Produktionsdaten haben sich in Bezug auf die Spirantisierung nach velarem Vokal drei Gruppen herausgebildet: Die GP aus SB und OF spirantisieren in der Vorleseaussprache (blau) so gut wie nicht. Die GP aus SW, OFL, NN und DT spirantisieren in der Vorleseaussprache unterschiedlich, die GP aus SW mehrheitlich häufig.

Wenn es sich bei der $g$-Spirantisierung um eine regiolektale Variante handelt, sollte man erwarten, dass die GP im wesentlichen autochthone Karten zeichnen, damit also anzeigen, dass diese Variante an ihrem Ort überhaupt gebraucht wird, unabhängig von der Situation.

Dies trifft für die GP aus SW und NN in hohem Maße zu, etwas weniger für OFL, OF und DT. In SB zeichnen alle drei GP aus Bad Saarow (BAS) allochthone, diejenigen aus Dahme autochthone Karten, ein Befund, der Anlass gibt, in anderen verfügbaren Daten der GP aus den beiden Erhebungsorten Hinweisen auf unterschiedliche Einstellungen und Orientierungen nachzugehen. Für mehr als die Hälfte der GP in den hier dargestellten Regionen hat die Spirantisierung die Dimension räumlicher Nähe, was auch mit der hohen Gebrauchshäufigkeit in informellen Kontexten korrespondiert. Diejenigen, die gleichfalls informell spirantisieren, aber allochthone Karten zeichnen, konstruieren ihren Erfahrungsraum nicht aus ihrer eigenen Sprachpraxis, sondern aus einer wie auch immer motivierten exklusiven oder exogenen Perspektive.

Die GP aus SW unterscheiden sich allerdings von allen anderen Regionen durch den Zusammenfall von Autochthonizität und häufiger Spirantisierung auch in der Vorleseaussprache

\footnotetext{
13 Von den mit \# markierten GP liegen keine Mental Maps vor, bei Markierungen mit * wurde das Tischgespräch auf Niederdeutsch geführt.
} 
(rote + blaue Balken), was darauf hindeutet, dass ihr Normhorizont hier regiolektal bestimmt sein dürfte.

Diejenigen GP, die autochthone Karten zeichnen, aber in der Vorleseaussprache nicht spirantisieren (NN, OFL, OF), orientieren sich eher an einem doppelten Normhorizont: für die Verwendung der spirantisierten Variante in informellen Kontexten am Regiolekt, für die Vermeidung dieser Variante in der Vorleseaussprache an der Standardsprache.

In SB und OF kartiert ein Teil der GP die Verbreitung der spirantisierten Variante in Arealen außerhalb des eigenen Ortes und verwendet sie ausschließlich in informellen Kontexten. Mithin dürfte es sich für diese GP hier um eine Variante ohne regiolektale Dimension handeln, deren Verwendung durch einen an der Standardsprache orientierten Normhorizont nur auf informelle Kontexte beschränkt ist. Um freilich ein zutreffendes Bild der individuellen Normhorizonte $\mathrm{zu}$ erhalten, muss auch die jeweilige Verortung der anderen Non-StandardMerkmale betrachtet werden. Das SiN-Korpus umfasst, wie anfangs beschrieben, Kartierungen $\mathrm{zu}$ sechs in allen Erhebungsregionen abgefragten Varianten (gefracht, Pabbe, Kinner, Kaffe, Kopp, dat), zusätzlich wurde die räumliche Verbreitung regionsspezifischer Formen abgefragt (dazu ausführlich Gessinger i. V.).

Es zeigt sich also bei einer differenzierteren datenbasierten Analyse, so mein Fazit, dass die Aussage, es handelte sich bei der $g$-Spirantisierung nach Velarvokal um ein weit verbreitetes norddeutsches Merkmal, zwar zutreffend ist, aber regionale Unterschiede in ihrer situativ gebundenen Realisierung und damit ihrem unterschiedlichen Status im Sprachlagenspektrum der Sprecherinnen überdeckt. Der SiN-Atlas kann diese Unterschiede auf Basis der Analyse der Produktionsdaten kartieren, die perzeptionslinguistischen Daten und Mental Maps machen den subjektiven Faktor sichtbar, der sich in diesen Unterschieden artikuliert. Komplementär zu der hier dargestellten regionsbezogenen Perspektive kann die Analyse sprecherbezogener soziobiographischer Daten und individueller Profile auf Grundlage phonetischer Abstandsmessungen ${ }^{14}$ Hinweise darauf geben, wie und in welchem Umfang usuelle regional- oder ortsgebundene Variation und individuelle Repertoires miteinander verschränkt sind.

\section{Literaturverzeichnis:}

Auer, Peter (1998): „Hamburger Phonologie. Eine variationslinguistische Skizze zur Stadtsprache der Hansestadt heute“. In: Zeitschrift für Dialektologie und Linguistik 65/2: 179_ 197.

Bieberstedt, Andreas/Ruge, Jürgen/Schröder, Ingrid (2016): „Kontaktinduzierte sprachliche Variation in der Hamburger Peripherie. Ein Modell zur Messung sprachlicher Konvergenz". In: Bieberstedt, Andreas/Ruge, Jürgen/Schröder, Ingrid (eds.): Hamburgisch. Struktur, Gebrauch, Wahrnehmung der Regionalsprache im urbanen Raum. Frankfurt a. M., Lang: 21-66. (= Sprache in der Gesellschaft 34).

Butterworth, Judith/Glawe, Meike (2011): „,Wir sprechen hier an und für sich reinrassiges Hochdeutsch“. Zur Erforschung der subjektiven Seite von Sprachverwendung“. In: Ganswindt, Brigitte/Purschke, Christoph (eds.): Perspektiven der Variationslinguistik. Beiträge

14 Cf. dazu Herrgen et al. (2001), Schröder 2011, Bieberstedt/Ruge/Schröder 2016. 
aus dem Forum Sprachvariation. Hildesheim/Zürich/New York, Olms: 371-393. (= Germanistische Linguistik 216/217).

Cassirer, Ernst (1990): Versuch über den Menschen. Einführung in eine Philosophie der Kultur. Frankfurt a. M.: Fischer.

Chambers, Jack K. (2009): Sociolinguistic Theory. Linguistic Variation and its Social Significance. Malden/MA: Wiley-Blackwell.

Dahl, Eva Sophie (1974): „Interferenz und Alternanz - zwei Typen der Sprachschichtenmischung im Norden der Deutschen Demokratischen Republik“. In: Ising, Gerhard (ed.): Aktuelle Probleme der sprachlichen Kommunikation. Berlin, Akademie-Verlag: 339-388.

Elmentaler, Michael/Gessinger, Joachim/Wirrer, Jan (2010): „Qualitative und quantitative Verfahren in der Ethnodialektologie am Beispiel von Salienz". In: Anders, Christina A./Hundt, Markus/Lasch, Alexander (eds.): Perceptual dialectology. Neue Wege der Dialektologie. Berlin/New York, de Gruyter: 112-153. (= Linguistik - Impulse und Tendenzen 38).

Elmentaler, Michael/Rosenberg, Peter (2015): Norddeutscher Sprachatlas (NOSA). Bd 1: Regiolektale Sprachlagen. Hildesheim/Zürich/New York: Olms.

Elmentaler, Michael et al. (2015): „Sprachvariation in Norddeutschland (SiN)“. In: Kehrein, Roland/Lameli, Alfred/Rabanus, Stefan (eds.): Regionale Variation des Deutschen. Projekte und Perspektiven. Berlin/Boston, de Gruyter: 397-424.

Garret, Peter/Coupland, Nikolas/Williams, Angie (2003): Investigating language attitudes. Social meaning of dialect, ethnicity and performance. Cardiff: University of Wales Press.

Gessinger, Joachim (2008a): „Ethnodialektologie und sprachlicher Wandel“. In: Stehl, Thomas (ed.): Kenntnis und Wandel der Sprachen. Beitrag zur Potsdamer Ehrenpromotion für Helmut Lüdtke. Tübingen, Narr: 57-79.

Gessinger, Joachim (2008b): „Perzeptive Dialektologie und Spracheinstellungsforschung“. Jahrbuch des Vereins für Niederdeutsche Sprachforschung 131: 133-145.

Gessinger, Joachim/Butterworth, Judith (2015): „Salienz als dynamisches interaktionales Konstrukt. Theoretische und methodische Aspekte“. In: Elmentaler, Michael/Hundt, Markus/Schmidt, Jürgen E. (eds.): Deutsche Dialekte: Konzepte, Probleme, Handlungsfelder. Akten des 4. Kongresses der Internationalen Gesellschaft für Dialektologie des Deutschen (IGDD) in Kiel. Stuttgart, Steiner: 259-294.

Gessinger, Joachim (i.V.): Varianz und Wahrnehmung: Der subjektive Faktor. Unter Mitarbeit von Judith Butterworth, Oliver Gondring und Mark Hillebrand. Hildesheim/Zürich/New York: Olms.

Gosztonyi, Alexander (1976): Der Raum. Geschichte seiner Probleme in Philosophie und Wissenschaften. Freiburg/München: Alber.

Herrgen, Joachim et al. (2001): Dialektalität als phonetische Distanz. Ein Verfahren zur Messung standarddivergenter Sprechformen. Marburg (Manuskript). www.deutschersprachatlas.de/publikationen/dialektalitaetsmessung. [28.07.2017].

Hettler, Yvonne (2013): ,,Nach Müllers geht man nicht! Zur Salienz und Bewertung morpho-syntaktischer Phänomene in Bremen und Hamburg“. In: Hettler, Yvonne et al. (eds.): Variation, Wandel, Wissen. Studien zum Hochdeutschen und Niederdeutschen. Frankfurt a. M., Lang: 161-183. (= Sprache in der Gesellschaft 32). 
Kehrein, Roland/Lameli, Alfred/Rabanus, Stefan (eds.) (2015): Regionale Variation des Deutschen. Projekte und Perspektiven. Berlin/Boston: de Gruyter.

Kiesewalter, Carolin (2011): „Zur Salienz remanenter Merkmale des Neuhessischen“. In: Ganswindt, Brigitte/Purschke, Christoph (eds.): Perspektiven der Variationslinguistik. Beiträge aus dem Forum Sprachvariation. Hildesheim/Zürich/New York, Olms: 335-369. (= Germanistische Linguistik 216/217).

Lambeck, Heino (1633): Düedsche Orthographia. Hamburg: Selbstverlag.

Lameli, Alfred (2015): „Zur Konzeptualisierung des Sprachraums als Handlungsraum“. In: Elmentaler, Michael/Hundt, Markus/Schmidt, Jürgen Erich (eds.): Deutsche Dialekte. Konzepte, Probleme, Handlungsfelder. Stuttgart, Steiner: 59-83. (=Zeitschrift für Dialektologie und Linguistik Beihefte 158).

Lenz, Alexandra (2010): „Zum Salienzbegriff und zum Nachweis salienter Merkmale“. In: Anders, Christina A./Hundt, Markus/Lasch, Alexander (eds.): Perceptual dialectology. Neue Wege der Dialektologie. Berlin/New York, de Gruyter: 89-111. (=Linguistik - Impulse und Tendenzen 38).

Mannheim, Karl (1980): Strukturen des Denkens. Frankfurt a. M.: Suhrkamp.

Paasi, Anssi (2003): "Region and Place: Regional identity in question". Progress in Human Geography 28/4: 475-485.

Purschke, Christoph (2011): Regionalsprachlichkeit und Hörerurteil. Grundzüge einer perzeptiven Variationslinguistik. Stuttgart: Steiner. (=Zeitschrift für Dialektologie und Linguistik Beihefte 149).

Purschke, Christoph (2015): "REACT - A constructivist theoretical framework for attitudes". In: Prikhodkine, Alexei/Preston, Dennis R. (eds.): Responses to Language Varieties. Variability, processes and outcomes. Amsterdam, Benjamins: 37-53. (=IMPACT. Studies in Language and Society 39).

Rácz, Péter (2013): Salience in Sociolinguistics. A quantitative approach. Berlin/Boston: de Gruyter.

Scharioth, Claudia (2015): Regionales Sprechen und Identität. Eine Studie zum Sprachgebrauch, zu Spracheinstellungen und Identitätskonstruktionen von Frauen in SchleswigHolstein und Mecklenburg-Vorpommern. Hildesheim/Zürich/New York: Olms. (= Deutsche Dialektgeographie 120).

Schmidt, Jürgen Erich/Herrgen, Joachim (2011) Sprachdynamik. Eine Einführung in die moderne Regionalsprachforschung. Berlin: Erich Schmidt.

Schröder, Ingrid (2011) „Dialekte im Kontakt. Individuelle Ausformungen des Sprachrepertoires“. In: Böhm, Manuela/Berner, Elisabeth/Erfurt, Jürgen (eds.): Nach dem linguistic turn. Sprachwissenschaft im Wandel. Duisburg, Universitätsverlag Rhein-Ruhr: 37-56. (= Osnabrücker Beiträge zur Sprachtheorie 78).

Schröder, Saskia (2013): „Zur Dynamik der /g/-Spirantisierung in den deutschen Regionalsprachen“. In: Hettler, Yvonne et al. (eds.): Variation, Wandel, Wissen. Studien zum Hochdeutschen und Niederdeutschen. Frankfurt a. M., Lang: 37-61. (= Sprache in der Gesellschaft 32).

Schröder, Saskia/Palliwoda, Nicole (2016): "Perceptual Dialectology - Speech Samples and the concept of Salience. Initial findings from the DFG-project ,Der deutsche Sprachraum aus der Sicht linguistischer Laien““. In: Cramer, Jennifer S./Montgomery, Chris (eds.). Cit- 
yscapes and Perceptual Dialectology: Global perspectives on non-linguists' knowledge of the dialect landscape. Berlin/Boston, de Gruyter: 257-274.

Siebs, Theodor (1930): Deutsche Bühnenaussprache: Hochsprache. Köln: Ahn.

Stellmacher, Dieter (1977): Studien zur gesprochenen Sprache in Niedersachsen. Eine soziolinguistische Untersuchung. Marburg: Elwert. (= Deutsche Dialektgeographie 82).

Ströker, Elisabeth (1965): Philosophische Untersuchungen zum Raum. Frankfurt a. M.: Klostermann.

Trudgill, Peter (1986): Dialects in Contact. Oxford etc.: Blackwell.

Viëtor, Wilhelm (1901): Die Aussprache des Schriftdeutschen. Leipzig: Reisland. 